\title{
Constraining the redshifts of unlocalised fast radio bursts ${ }^{\star}$
}

\author{
Charles R. H. Walker ${ }^{1}$, Yin-Zhe Ma ${ }^{2,3,1}$, and René P. Breton ${ }^{1}$ \\ ${ }^{1}$ Jodrell Bank Center for Astrophysics, The University of Manchester, Alan Turing Building, Manchester, M13 9PL, UK \\ e-mail: charles.walker@postgrad.manchester.ac.uk; rene.breton@manchester.ac.uk \\ ${ }^{2}$ School of Chemistry and Physics, University of KwaZulu-Natal, Westville Campus, Private Bag X54001, Durban 4000, \\ South Africa \\ e-mail: ma@ukzn.ac.za \\ ${ }^{3}$ NAOC-UKZN Computational Astrophysics Center (NUCAC), University of KwaZulu-Natal, Durban 4000, South Africa
}

Received 4 April 2018 / Accepted 20 January 2020

\begin{abstract}
Context. The relationship between the dispersion measures (DMs) and redshifts of fast radio bursts (FRBs) is of scientific interest. Upcoming commensal surveys may detect and localise many FRBs to the sub-arcsecond angular resolutions required for accurate redshift determination. Meanwhile, it is important to exploit sources accumulated with more limited localisation to their maximum scientific potential.

Aims. We present techniques for the DM-redshift analysis of large numbers of unlocalised FRBs, accounting for uncertainties due to their extragalactic DM components, redshift dependences, and progenitor scenarios.

Methods. We reviewed the components comprising observed FRB DMs. We built redshift-scalable probability distribution functions for these components, which we combined in cases of multiple progenitor scenarios. Accounting for prior FRB redshift distributions we inverted these models, enabling FRB redshifts to be constrained.

Results. We illustrate the influence of FRB progenitors on their observed DMs, which may remain significant to redshift $z \sim 3$. We identify the FRB sample sizes required to distinguish between multiple progenitor scenarios. We place new, physically motivated redshift constraints on all catalogued FRBs to date and use these to reject potential host galaxies in the localisation area of an FRB according to various models. We identify further uses for DM-redshift analysis using many FRBs. We provide our code so that these techniques may be employed using increasingly realistic models as our understanding of FRBs evolves.
\end{abstract}

Key words. techniques: miscellaneous - stars: neutron - pulsars: general - local insterstellar matter - galaxies: halos intergalactic medium

\section{Introduction}

Many fast radio bursts (FRBs) have unconstrained redshifts. Since their discovery in 2007 (Lorimer et al. 2007), tens of these short-duration $(\sim 0.1-10 \mathrm{~ms})$, dispersed radio bursts have been catalogued (Petroff et al. 2016) ${ }^{1}$. Save for the repeating FRB 121102 (Spitler et al. 2014, 2016), their extragalactic origins have been inferred via their dispersion measures (DMs).

These DMs, related to the frequency-dependent index of refraction and resulting arrival time delays experienced by radio waves which propagate through ionised electrons, are proportional to the integrated electron densities along their propagation paths. All FRBs have DMs measured in excess of that which may be afforded by electrons of the Milky Way alone.

Extragalactic electrons may lie in the intergalactic medium (IGM), the host galaxy of the FRB and the environment around the source (Macquart et al. 2015). According to relativistic theories, the observed time delay associated with these electrons is modified. This, along with sightline variance in IGM electrons within collapsed structures results in a redshift-dependent potential DM distribution for FRBs (Ioka 2003; McQuinn 2014; Macquart et al. 2015). This distribution is additionally intrinsically linked to the locations of FRBs within their host galaxies and thus to their progenitors.

\footnotetext{
* Python code and examples are available at https://doi.org/10. 5281/zenodo. 1209920

1 http://www. frbcat.org
}

Analysis of the FRB DM-redshift relationship is of astronomical significance. Macquart et al. (2015) review the scientific potential of redshift-constrained FRBs, including using $10^{4}$ as probes of the baryonic matter of the Universe and $10^{3}$ as cosmic rulers for measuring the geometry of the Universe beyond redshift $z \sim 2$. Statistical studies into the potential IGM (see Zhou et al. 2014; McQuinn 2014) and host galaxy (see Xu \& Han 2015; Yang et al. 2017) contributions to FRB DMs have been undertaken to compare different models accounting for the structure of the IGM or the spacial distributions of FRBs within host galaxies, for example. In practice, many FRBs with well-determined redshifts must be accumulated to exploit such studies fully.

Redshifts may be obtained spectroscopically via host galaxy association using interferometry (e.g. Tendulkar et al. 2017), however many FRBs have been (and will continue to be) detected by telescopes with large fields of view (FoVs) containing many galaxies, within which their locations may be unconstrained. The FoV of a Parkes $L$-band multi-beam receiver (half power beam width; $H P B W \sim 14.4^{\prime}$ ), for example, may contain 65000 galaxies out to redshift 6 (Scoville et al. 2007) ${ }^{2}$. Molonglo's UTMOST may improve localisation to $15^{\prime \prime} \times 15^{\prime \prime}$ (Caleb et al. 2017); ASKAP's fly's-eye mode has demonstrated localisation of an FRB to $8^{\prime} \times 8^{\prime}$ (Bannister et al. 2017) and aims for $<7^{\prime \prime}$ localisation when fully operational (Macquart et al. 2010); and CHIME will improve localisation for potentially

2 As expected by COSMOS 2 , to a limiting magnitude $I_{\mathrm{AB}}(10 \sigma)<$ 27.5.

A37, page 1 of 14 
tens of FRBs per day to tens of arcminutes ( $\mathrm{Ng}$ et al. 2017; CHIME/FRB Collaboration 2018). But the large FoVs and onsky time available to resolution-limited, single dish and fly's-eye surveys suggest that many FRBs will be discovered to worse than the sub-arcsecond accuracy necessary for unambiguous association with a single host (see e.g. Mahony et al. 2018).

Standard calculation of redshifts for such unlocalised FRBs involves estimation and removal of the sightline-dependent DM component attributed to Milky Way electrons (using Galactic electron models, e.g. NE2001, Cordes \& Lazio 2002 or YMW, Yang et al. 2017). This may be followed by simplification of the theoretical relationship (see Ioka 2003) between redshift and the IGM DM component (see e.g. Petroff et al. 2016), assuming idealised hydrogen and helium ionisation fractions and an average line of sight. Finally, no host DM component (e.g. Petroff et al. 2016) or a fixed host component (e.g. $\sim 100 \mathrm{pc} \mathrm{cm}^{-3}$; Lorimer et al. 2007; Thornton et al. 2013) may be assumed. Associated uncertainties from, for example redshift-dependent variance in collapsed systems (e.g. galactic haloes) along IGM sightlines (potentially contributing $\sim 180-400 \mathrm{pc} \mathrm{cm}^{-3}$ at redshift 1 ; McQuinn 2014) or source positions in host galaxies (potentially contributing thousands of $\mathrm{pc} \mathrm{cm}^{-3}$ in the rest frame, $\mathrm{Xu} \& \mathrm{Han}$ 2015; Yang et al. 2017), may not be considered.

These uncertainties leave many catalogued FRBs with redshifts that are not ideally constrained, which hinders analysis of the DM-redshift relationship. The purposes of this paper are as follows: Firstly, we aim to introduce a framework for performing physically motivated DM-redshift analysis for large numbers of unlocalised FRBs, accounting for uncertainties such as (a) potential host contributions to DM (following e.g. Xu \& Han 2015; Yang et al. 2017), (b) the cosmic variance of IGM structure (following e.g. McQuinn 2014), and (c) FRB progenitor evolution across cosmic time (following e.g. Zhou et al. 2014). Secondly, we apply physically motivated models to this framework, thus illustrating the effects of different progenitor scenarios on the probability distribution functions (PDFs) of observed DMs and redshifts of FRBs. Lastly, we discuss the implications of our models, to provide constrained redshift values for currently unlocalised FRBs and to identify further uses for FRB DM-redshift analysis.

In Sect. 2 we summarise the extragalactic DM components which contribute to the observed arrival time delay between two FRB frequencies and review their respective redshift dependences. We discuss our choices for physically motivated PDFs of these DM components. In Sect. 3 we present a framework for the combination of these PDFs to retrieve the overall likelihood of an FRB of given redshift having a particular excess DM, or vice versa. We demonstrate this method using our models. Section 4 discusses the implications of our findings.

Unless otherwise stated, in this paper we adopt a spatially flat $\Lambda \mathrm{CDM}$ cosmology model with the cosmological parameters fixed to the best-fit values of Planck Collaboration VI (2020): $\Omega_{\mathrm{b}}=0.048, \Omega_{\mathrm{m}}=0.309, \Omega_{\Lambda}=0.691, n_{\mathrm{s}}=0.9608, \sigma_{8}=0.815$, $H_{0}=67.3 \mathrm{~km} \mathrm{~s}^{-1} \mathrm{Mpc}^{-1}$.

\section{Contributions to FRB dispersion measure}

The observed time delay $\Delta t_{\text {obs }}$ between two frequencies $v_{1, \mathrm{obs}}$ and $v_{2, \text { obs }}$ may be written as a function of dispersion measure $\mathrm{DM}_{\mathrm{obs}}$ (Manchester \& Taylor 1972). For an extragalactic FRB, DM may be deconstructed into components

$\mathrm{DM}_{\mathrm{obs}}=\mathrm{DM}_{\mathrm{MW}}+\mathrm{DM}_{\mathrm{IGM}}+\mathrm{DM}_{\text {host }}+\mathrm{DM}_{\mathrm{local}}$, representing contributions from electrons along the FRB light path in the MW, IGM, its host galaxy, and its local environment (dependent on its progenitor model), respectively (Macquart et al. 2015). The excess extragalactic contribution to $\mathrm{DM}_{\mathrm{obs}}$ (i.e. $\left.\mathrm{DM}_{\mathrm{IGM}}+\mathrm{DM}_{\text {host }}+\mathrm{DM}_{\text {local }}\right)$ is referred to in this work as $\mathrm{DM}_{\mathrm{exc}}$.

\subsection{Milky Way}

For a given FRB, DM $\mathrm{MW}_{\mathrm{MW}}$ is typically computed using Galactic electron distributions (e.g. NE2001 Cordes \& Lazio 2002, YMW Yang et al. 2017) modelling the Milky Way interstellar medium (ISM). A contribution from the Galactic halo of the Milky Way (estimations in the range from $2-30 \mathrm{pc} \mathrm{cm}^{-3}$ as reviewed by Mahony et al. 2018) may also be considered. Removal of a completely accurate $\mathrm{DM}_{\mathrm{MW}}$ leaves a remaining $\mathrm{DM}_{\mathrm{exc}}$ component solely associated with extragalactic electron distributions. Uncertainties associated with Milky Way electron models may be considered (e.g. in Keane 2016). Through analysis of pulsars with parallax-determined distances, the potential of such models to misrepresent the $\mathrm{DM}_{\mathrm{MW}}$ contribution to $\mathrm{DM}_{\mathrm{obs}}$ has been demonstrated, the most extreme result of which could be the misclassification of an FRB as another transient phenomenon, or vice versa. In particular Keane (2016) highlight a source, $\mathrm{J} 1354+24$, as having a 20-40\% probability of being an FRB wrongly labelled as an RRAT. For the purposes of this paper we assume correct $\mathrm{DM}_{\mathrm{MW}}$ values and discard these to focus on extragalactic, redshift-dependent contributions to uncertainty (see Sect. 4.1 for a discussion).

\subsection{Intergalactic medium}

While $40 \%$ of the baryonic matter budget of the Universe is readily accounted for (e.g. in galaxies, X-ray corona, and warm intergalactic matter), the remaining portion is less easily detectable. Recent use of the thermal Sunyaev-Zel'dovich effect (e.g. de Graaff et al. 2019; Tanimura et al. 2019) and quasar absorption line observations (e.g. Nicastro et al. 2018) reveal large-scale filamentary structure in the IGM as a potential source of this remaining matter. As all IGM electrons along an FRB propagation path contribute to $\mathrm{DM}_{\mathrm{IGM}}$, FRBs may similarly serve as probes of elusive baryon distributions (McQuinn 2014).

The DM $\mathrm{IGM}_{\mathrm{IG}}$ is source redshift $\left(z_{\mathrm{s}}\right)$ dependent. During standard $\mathrm{DM}_{\mathrm{IGM}}$-redshift analysis, an ionisation fraction profile for the IGM is assumed, accounting for the reionisation history of both hydrogen and helium ${ }^{3}$. A linear approximation to the resulting $\left\langle\mathrm{DM}_{\mathrm{IGM}}\left(z_{\mathrm{s}}\right)\right\rangle$ profile, which is the mean IGM contribution to FRB DMs as a function of redshift when averaged over all sightlines, is then used to estimate $z_{\mathrm{s}}$ for observed FRBs. These linear relationships take the form $z_{\mathrm{s}}=\left(\mathrm{DM}_{\mathrm{obs}}-\mathrm{DM}_{\mathrm{MW}}\right) / \mathrm{X} \mathrm{pc} \mathrm{cm}{ }^{-3}$, where $\mathrm{X}$ is constant and dependent on the exact ionisation fractions assumed (see e.g. Petroff et al. 2016; Keane 2018; Zhang 2018). Additionally, sightline variability in the number of collapsed systems (e.g. galactic haloes) along FRB propagation paths results in a variance around $\left\langle\mathrm{DM}_{\mathrm{IGM}}\left(z_{\mathrm{s}}\right)\right\rangle$ for an FRB population (Ioka 2003; McQuinn 2014). To accurately contain FRB DM-redshift constraints, this variance must be considered.

McQuinn (2014) simulate PDFs describing the likelihood of an FRB originating at source redshift $z_{\mathrm{s}}$ contributing an IGM component $\mathrm{DM}_{\mathrm{IGM}}$ to the total observed $\mathrm{DM}_{\mathrm{obs}}$, according to various galactic halo electron distributions. Following this work, we consider a Gaussian approximation $P\left(\mathrm{DM}_{\mathrm{IGM}} \mid z_{\mathrm{s}}\right)$ for this

3 The true ratio of ionised to total electrons may be between 0.86 and 1 (see Appendix A). 


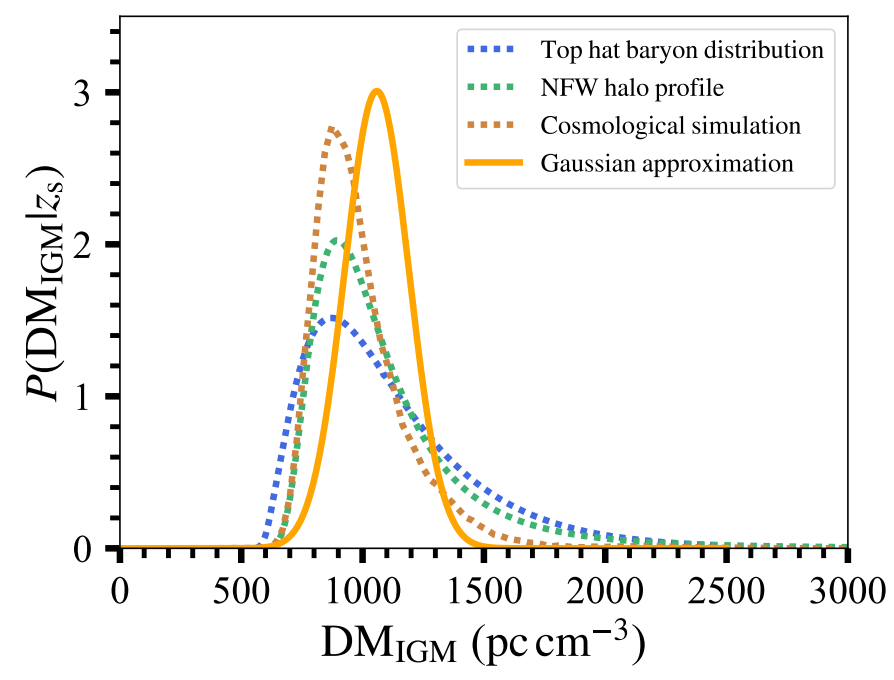

Fig. 1. Probability distribution functions $\left(P\left(\mathrm{DM}_{\mathrm{IGM}}\right)\right)$ for $\mathrm{FRBs}$ of source redshift $z_{\mathrm{s}}=1$ accounting for $\mathrm{DM}_{\mathrm{IGM}}$ sightline variance due to collapsed systems. The Gaussian approximation assumed in this work is compared to more complex McQuinn (2014) baryonic halo models (tracing top hat functions, NFW profiles, and cosmological simulations, respectively, obtained via. private communication). Profiles are normalised to $\int P\left(\mathrm{DM} \mid z_{\mathrm{s}}=1\right) \mathrm{d} \mathrm{DM}=1000$.

distribution with redshift-evolving mean $\operatorname{DM}_{\mathrm{IGM}}\left(z_{\mathrm{s}}\right)$ and variance $\sigma_{\mathrm{DM}}^{2}\left(\mathrm{DM}, z_{\mathrm{s}}\right)$. We make use of the matter power spectrum from public code $\mathrm{CAMB}^{4}$, with Planck best-fitting cosmological parameters (Planck Collaboration VI 2020). More complex profiles may be substituted in the future. Our approximation, derived in full in Appendix A, is shown in Fig. 1.

\subsection{Host galaxy}

As discussed by, for example Ioka (2003), Zhou et al. (2014) and Macquart et al. (2015), the arrival time delay $\Delta t_{\text {obs,host }}$ between observing frequencies $v_{1, \text { obs }}$ and $v_{2, \text { obs }}$ for an FRB of source redshift $z_{\mathrm{s}}$ due to a rest-frame host DM contribution $\mathrm{DM}_{\text {host,r }}$ depends on the following:

1. The relationship between the frequency of the radio wave as emitted in the rest frame of a receding galaxy $v_{\mathrm{r}}$ and the observed frequency $v_{\mathrm{obs}}$

$$
\frac{v_{\mathrm{r}}}{v_{\mathrm{obs}}}=\left(1+z_{\mathrm{s}}\right)
$$

2. The relationship between a time delay measured in the rest frame of the galaxy $\Delta t_{\mathrm{r}, \text { host }}$ and the observed $\Delta t_{\mathrm{obs} \text {, host }}$ due to cosmological time dilation,

$$
\frac{\Delta t_{\mathrm{obs}, \text { host }}}{\Delta t_{\mathrm{r}}}=\left(1+z_{\mathrm{s}}\right)
$$

such that

$\Delta t_{\text {obs,host }} \propto \mathrm{DM}_{\text {host }}\left(z_{\mathrm{s}}\right)\left(\frac{1}{v_{1, \mathrm{obs}}^{2}}-\frac{1}{v_{2, \mathrm{obs}}^{2}}\right)$,

where

$\operatorname{DM}_{\text {host }}\left(z_{\mathrm{s}}\right)=\frac{\mathrm{DM}_{\text {host, }}}{1+z_{\mathrm{s}}}$

4 https://camb.info
The $\mathrm{DM}_{\text {host }}$ contributions to $\mathrm{DM}_{\mathrm{obs}}$ have previously been investigated and found to be dependent on both FRB host galaxy types and the source locations within them. Ioka (2003) explored host galaxy components for GRBs, finding potential contributions of up to $10^{5} \mathrm{pc} \mathrm{cm}^{-3}$ for sources in star-forming regions. Xu \& Han (2015) evaluate FRB DM host $_{\text {values from rest-frame }}$ spiral and elliptical galaxies by scaling NE2001, finding potential contributions of approximately thousands/tens of $\mathrm{pc} \mathrm{cm}^{-3}$, respectively. Yang et al. (2017) estimate the mean host contribution for 21 observed FRBs to be $\sim 270 \mathrm{pc} \mathrm{cm}^{-3}$. Accounting for $\mathrm{DM}_{\text {host }}$ variation during DM-redshift analysis (particularly of low $z_{\mathrm{s}}$ events) is therefore necessary.

In this work we simulate rest-frame $\left(z_{\mathrm{s}}=z_{0}=0\right)$ host galaxy electron distribution models, which we populate with FRBs according to various progenitor scenarios. By integrating electron densities along random sightlines we build rest-frame PDFs describing the likelihood of host galaxies contributing $\mathrm{DM}_{\text {host }}$ components to $\mathrm{DM}_{\mathrm{obs}}$. For demonstrative purposes, we model static galaxies which do not evolve with redshift ${ }^{5}$. By letting $P(U) \mathrm{d} U=P(x) \mathrm{d} x$ with $\mathrm{DM}_{\text {host }}=U(x)$ and $\mathrm{DM}_{\text {host } \mathrm{r}}=x$ the redshift-dependent PDF becomes

$P\left(\mathrm{DM}_{\text {host }} \mid z=z_{\mathrm{s}}\right)=\left(1+z_{\mathrm{s}}\right) P\left(\left(1+z_{\mathrm{s}}\right) \mathrm{DM}_{\text {host }} \mid z=z_{0}\right)$.

Our models are described below.

\subsubsection{Spiral galaxies}

As simple spiral galaxy electron distribution models (see Schnitzeler 2012 for a review) have previously been proven sufficient to reproduce the DMs of Milky Way pulsars with known parallax distances to within a factor 1.5-2, rather than scaling NE2001 (see e.g. Xu \& Han 2015) to demonstrate our DM-redshift analysis framework, we opt for a simpler model comprised of two components. The first is the GBCa model (as denoted by Schnitzeler 2012), which was developed using 109 pulsars with DM-independently determined distances Gómez et al. (2001). This model is a sum of two ellipsoids made from decaying exponentials and describes the inner Milky Way Galactic disc, where the majority of the stellar mass of the Milky Way resides. It is well constrained within 4$12 \mathrm{kpc}$ of the Galactic centre. We make the assumption that the model is acceptable within these central $4 \mathrm{kpc}$ for the demonstrative purposes of this work. The second component is a model tracing neutral hydrogen (HI), which was developed by Kalberla \& Dedes (2008). This model is a single decaying exponential and approximates the average mid-plane volume density of the Milky Way disc between 7 and $35 \mathrm{kpc}$. We combine these two components into a smoothly varying function as follows:

$n_{\mathrm{e}, \mathrm{spiral}}(r, h)=\frac{1}{1+\exp \left(\left(r-r_{f}\right) / r_{n}\right)} \sum_{i=1}^{2} n_{i} \frac{\exp \left(-r / h_{r, i}\right)}{\exp \left(-r_{\odot} / h_{r, i}\right)} \exp \left(\frac{-h}{h_{z, i}}\right)$,

where cylindrical coordinates $r$ and $h$ denote the radial distance from the Galactic centre and height above the Galactic plane, respectively. All relevant parameters are provided in Table 1. To reconcile the two components, the HI model is multiplied by an ionisation fraction of $\sim 15 \%$. At a radius $\sim 50 \mathrm{kpc}$ the model reaches an electron density consistent with the IGM at redshift zero as estimated by Ioka (2003). The model is shown in Fig. 2.

\footnotetext{
5 See for example Luo et al. (2018) for an approach to galaxy simulation accounting for a mass ranges and evolution with redshift.
} 

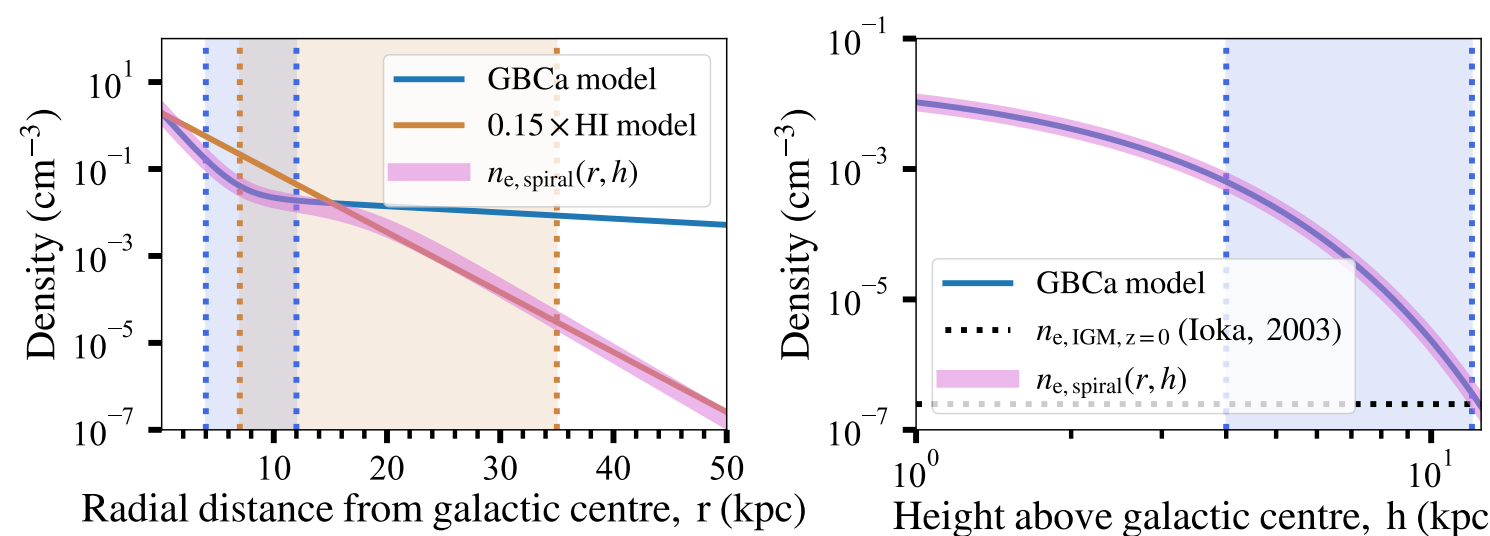

Fig. 2. Radial and height cross sections of our spiral galaxy electron density model $n_{\text {e,spiral }}(r, h)$ (pink lines). Constituent components of the model tracing the inner MW Galactic disc (Gómez et al. 2001; Schnitzeler 2012) (blue lines) and an ionised outer HI disc model (Kalberla \& Dedes 2008) are shown (brown lines). Shaded (blue/brown) regions highlight areas in which the inner/outer profiles are considered well constrained. The horizontal black dotted line indicates the approximate electron density for the IGM at $z_{\mathrm{s}}=0$ (Ioka 2003).

Table 1. Spiral galaxy electron distribution model parameters.

\begin{tabular}{ll}
\hline \hline Parameter & Value \\
\hline$h_{r, 1}$ & $30.4 \mathrm{kpc}$ \\
$h_{r, 2}$ & $1.5 \mathrm{kpc}$ \\
$h_{z, 1}$ & $1.07 \mathrm{kpc}$ \\
$h_{z, 2}$ & $0.050 \mathrm{kpc}$ \\
$n_{1}$ & $2.03 \times 10^{-2} \mathrm{~cm}^{-3}$ \\
$n_{2}$ & $0.71 \times 10^{-2} \mathrm{~cm}^{-3}$ \\
$r_{f}$ & $17.5 \mathrm{kpc}$ \\
$r_{n}$ & $3.15 \mathrm{kpc}$ \\
\hline
\end{tabular}

References. See Gómez et al. (2001), Schnitzeler (2012), Kalberla \& Dedes (2008).

\subsubsection{Elliptical galaxies}

We use a $\beta$ model to describe elliptical galaxy electron distributions, i.e.

$n_{\mathrm{e}, \text { elliptical }}(r)=F * \rho_{0}\left(1+\left(\frac{r}{r_{\mathrm{c}}}\right)^{2}\right)^{-3 \beta_{\mathrm{g}} / 2}$,

following Brown \& Bregman (2001), Mamon \& Łokas (2005). The model traces hot gas density within the virial radius as a function of galactrocentric radius $r$, central density $\rho_{0}, \beta_{\mathrm{g}}=0.5$, and $r_{\mathrm{c}}$ as follows:

$r_{\mathrm{c}} \simeq \frac{R_{\mathrm{e}}}{q}$

where $R_{\mathrm{e}}$ is the effective (half-light) galaxy radius and $q=10$. Assuming hot gas traces free electrons, we modify the model to approach the local IGM electron density at the virial radius of a galaxy $(\sim 70 \mathrm{kpc})$ via multiplication with an ionisation fraction $(F=0.04)$. The relationships between total galaxy mass, central density, and effective radius for the $\beta$ model are provided by Mamon \& Łokas (2005). We model a galaxy of total virial mass $5 \times 10^{10} M_{\odot}$ with $\rho_{0} \simeq 6 \times 10^{7} M_{\odot} \mathrm{kpc}^{-3}$ and $R_{\mathrm{e}}=3.2 \mathrm{kpc}$.

\subsubsection{Distributions of FRBs within galaxies}

Spacial distributions of FRBs within host galaxies rely on their progenitors. To model potential scenarios we populate our host galaxies with FRBs of different $x, y, z$ coordinates and calculate DMs for randomly orientated observers at their boundaries. The resulting $P\left(D M_{\text {host }} \mid z_{\mathrm{s}}=0\right)$ PDFs are shown in Fig. 3. Our modelling choices are discussed below.

Stellar distributions in spiral galaxies. Stellar populations and phenomena potentially associated with FRBs include massive progenitor stars and superluminous supernovae (Tendulkar et al. 2017), magnetar outbursts (Pen \& Connor 2015), pulsar supergiant pulses (Cordes \& Wasserman 2016), collapsing (Falcke \& Rezzolla 2014) or merging (Totani 2013) neutron stars, and pulsar companions (Mottez \& Zarka 2014). Such progenitors should follow the spacial distributions of young stellar populations (e.g. OB stars) or neutron stars. We opted to model OB stars, pulsar populations of two ages (hereafter YPSRs, OPSRs) separated by a 8 Myr characteristic age, and millisecond pulsars (MSPs) separately to account for displacement due to, for example, supernova kicks.

Height distributions as a function of height $h$ above the galactic plane $\left(H_{*}(h)\right.$, where * denotes the model type) for OB stars, YPSRs, and OPSRs are modelled following Maíz-Apellániz (2001); Sun \& Han (2004) and written as

$$
\begin{aligned}
& H_{\mathrm{OB}}(h)=\frac{1}{\sqrt{2 \pi} h_{\mathrm{OB}}} \exp \left(-\frac{h^{2}}{2 h_{\mathrm{OB}}^{2}}\right), \\
& H_{\mathrm{YPSR}}(h)=\frac{2}{\sqrt{2 \pi} h_{\mathrm{PSR}}} \exp \left(-\frac{h^{2}}{2 h_{\mathrm{PSR}}^{2}}\right), \\
& H_{\mathrm{OPSR}}(h)=\frac{2}{\sqrt{2 \pi} h_{\mathrm{PSR}}} \exp \left(-\frac{h^{2}}{2 h_{\mathrm{PSR}}^{2}}\right)+\frac{1}{h_{\mathrm{OPSR}}} \exp \left(\frac{|h|}{h_{\mathrm{OPSR}}}\right),
\end{aligned}
$$

where the scale height $h_{\mathrm{PSR}}=h_{0}+\sigma_{\mathrm{PSR}} t$. The height distribution for MSPs is modelled after Lorimer (2013) as follows:

$H_{\mathrm{MSP}}(h)=\frac{1}{h_{\mathrm{MSP}}} \exp \left(-\frac{h}{h_{\mathrm{MSP}}}\right)$.

Parameters are provided in Table 2.

Radial distributions as a function of galactocentric radius $\left(R_{*}(r)\right.$, where $*$ denotes the model type) for YPSRs and OPSRs, are modelled with a gamma function following Yusifov \& Küçük (2004) and with a Gaussian distribution for MSPs and OB stars following Lorimer (2013) and written as

$R_{\mathrm{YPSR}}(r)=R_{\mathrm{OPSR}}(r)=A_{\mathrm{PSR}}\left(\frac{X}{X_{\odot}}\right)^{a} \exp \left(-b\left(\frac{X-X_{\odot}}{X_{\odot}}\right)\right)$, 

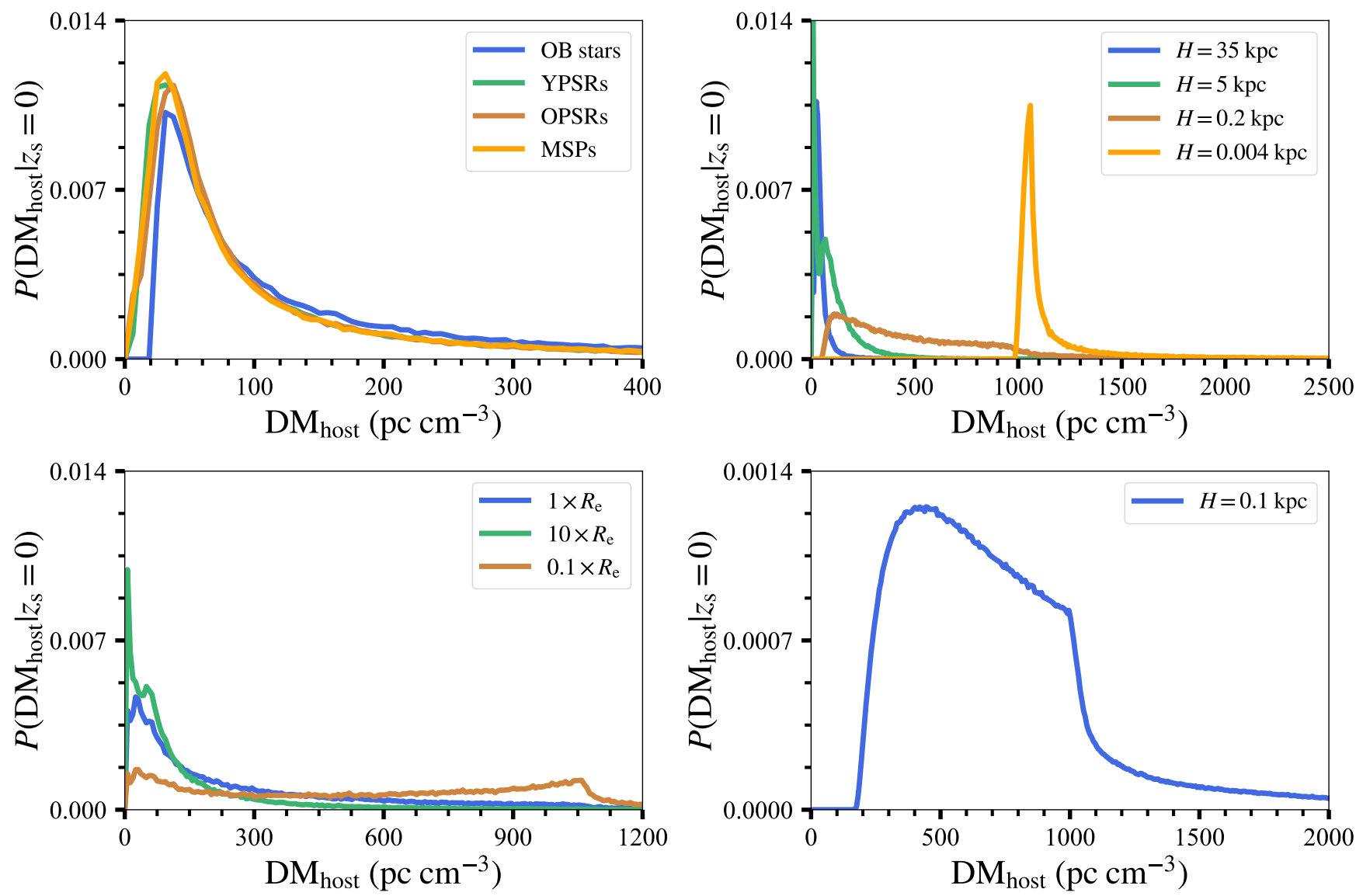

Fig. 3. Rest-frame PDFs of relative likelihood against $\mathrm{DM}_{\text {host }}$ for multiple FRB progenitor scenarios. From top left to bottom right: sources follow stellar distributions (OB stars, YPSRs, OPSRs, MSPs) in spiral galaxies; homogeneous distributions (within spheres of radius $H \mathrm{kpc}$ ) in spiral galaxies; elliptical distributions (confined to multiples of the galaxy effective radius, $R_{\mathrm{e}}=3.2 \mathrm{kpc}$ ) in elliptical galaxies; and homogeneous distributions (within spheres of radius $H \mathrm{kpc}$ ) in elliptical galaxies.

Table 2. Parameters used in pulsar distribution models.

\begin{tabular}{ll}
\hline \hline Parameter & Value \\
\hline$\sigma$ & $7.5 \mathrm{kpc}$ \\
$r_{\odot}$ & $8.5 \mathrm{kpc}$ \\
$r_{1}$ & $0.55 \pm 0.10 \mathrm{kpc}$ \\
$A_{\mathrm{PSR}}$ & $1.64 \pm 0.11$ \\
$a$ & $1.64 \pm 0.11$ \\
$b$ & $4.01 \pm 0.24$ \\
$h_{\mathrm{OB}}$ & $63 \mathrm{pc}$ \\
$h_{0}$ & $49 \times 10^{-3} \mathrm{kpc}$ \\
$\sigma_{\mathrm{PSR}}$ & $282 \mathrm{~km} \mathrm{~s}$ \\
$h_{\mathrm{OPSR}}$ & $0.4 \mathrm{kpc}$ \\
$t$ (YPSR) & $2 \mathrm{Myr}$ \\
$t$ (OPSR) & $1 \mathrm{Gyr}$ \\
$h_{\mathrm{MSP}}$ & $0.5 \mathrm{kpc}$ \\
\hline
\end{tabular}

References. Yusifov \& Küçük (2004), Sun \& Han (2004), Maíz-Apellániz (2001).

where $X=r+r_{1}$ and $X_{\odot}=r_{\odot}+r_{1}$.

$R_{\mathrm{OB}}(r)=R_{\mathrm{MSP}}(r)=\frac{1}{\sqrt{2 \pi} \sigma} \exp \left(-\frac{r^{2}}{2 \sigma^{2}}\right)$.

Parameters are provided in Table 2.
Other distributions. We populate elliptical galaxies assuming their stellar mass distribution (and thus ionised electron distribution) to be a tracer for FRBs. We achieve this with a source distribution, following $n_{\mathrm{e}, \text { elliptical }}(r)$ normalised to the number simulated sources. We also generate homogeneously distributed sources within spheres of variable radius for both spiral and elliptical galaxies. Such distributions attempt to model central galactic halo-based progenitors. At smaller limiting radii these distributions could serve as models of AGN-related FRB mechanisms (e.g. relativistic jet-plasma interactions Romero et al. 2016; Vieyro et al. 2017). Larger limiting radii could be consistent with progenitors located in more extended galactic haloes. We note that the most accurate DM distributions at the smallest limiting radii require electron distribution models with well-defined central profiles.

\subsection{Burst environment}

The $\mathrm{DM}_{\text {local }}$ contributions to $\mathrm{DM}_{\mathrm{obs}}$ are also progenitordependent. Progenitor theories of FRB are diverse and may be separated into cataclysmic and non-cataclysmic events. The repeating FRB 121102, by virtue of its repetition, must be noncataclysmic. Sub-arcsecond localisation has enabled study of its environment. This object is hosted by a low-metallicity dwarf (Tendulkar et al. 2017) and is associated with a compact, persistent, off-centre (with respect to the optical centre of the galaxy) variable radio source within a star-forming region, which may 
be a low-luminosity AGN that is powered supernova remnant or pulsar wind nebula (Chatterjee et al. 2017; Marcote et al. 2017; Bassa et al. 2017). The extreme and variable rotation measures of the burst indicate possible proximity of a neutron star and massive accreting black hole, and parallels to PSR J1745-2900 and Sagittarius A* have been drawn (Michilli et al. 2018) ${ }^{6}$. Analysis

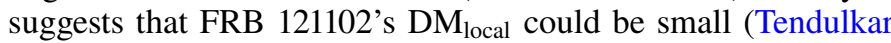
et al. 2017). Such studies have potential implications for progenitor models, however FRB 121102 may not necessarily be representative of all FRBs. More accurate modelling of $\mathrm{DM}_{\text {local }}$ may arise as further FRBs are localised. In this work we assume $\mathrm{DM}_{\text {local }}=0$.

\section{Excess electron model}

In this section we present the framework for combining the PDFs of $\mathrm{DM}_{\mathrm{exc}}$ components to retrieve a PDF, which describes the likelihood of an FRB at source redshift $z_{\mathrm{s}}$ to have an observed extragalactic DM component $\mathrm{DM}_{\mathrm{exc}}$, contributing to a measured time delay $\Delta t_{\mathrm{obs} \text {,exc }}$ between two observing frequencies $v_{1, \mathrm{obs}}$ and $v_{2, \mathrm{obs}}$ on Earth. We demonstrate this using our simulated IGM and progenitor models. Increasingly, accurate component models developed using FRB discoveries (or via other means) may allow these PDFs, which we refer to as excess electron models, to become increasingly useful tools for DM-redshift analysis. In Sect. 3.1 we describe our method and in Sect. 3.2 we apply Bayes' theorem to the excess electron model and discuss advantages of doing so.

\section{1. $P(D M / z)$ methodology and results}

Following Eq. (1), an FRB emitted from a source at redshift $z_{\mathrm{s}}$ has a $\mathrm{DM}_{\mathrm{exc}}$ formed from the sum of its extragalactic components. Assuming these components to be random independent variables drawn from their respective underlying PDFs, $P\left(\mathrm{DM}_{\mathrm{IGM}} \mid z_{\mathrm{s}}\right)$ and $P\left(\mathrm{DM}_{\text {host }} \mid z_{\mathrm{s}}\right)$, the convolution of these PDFs,

$P\left(\mathrm{DM}_{\mathrm{exc}} \mid z_{\mathrm{s}}\right)=P\left(\mathrm{DM}_{\mathrm{IGM}} \mid z_{\mathrm{s}}\right) * P\left(\mathrm{DM}_{\text {host }} \mid z_{\mathrm{s}}\right)$

describes the likelihood of an FRB of given source redshift $z_{\mathrm{s}}$ to have an observed extragalactic DM component $\mathrm{DM}_{\mathrm{exc}}$. This $\mathrm{DM}_{\text {exc }}$ results in a measured time delay $\Delta t_{\mathrm{obs} \text {,exc }}$ between two observing frequencies $\left(v_{1, \mathrm{obs}}\right.$ and $\left.v_{2, \mathrm{obs}}\right)$ on Earth.

Example excess electron models generated using our IGM (Sect. 2.2) and host galaxy (Sect. 2.3) PDFs are shown in Fig. 4. Comparisons between projections of these models for different values of $z_{\mathrm{s}}$ and $P\left(\mathrm{DM}_{\mathrm{IGM}} \mid z_{\mathrm{s}}\right)$ illustrate the initially significant (and diminishing with redshift) influence of the host galaxy on $P\left(\mathrm{DM}_{\mathrm{exc}} \mid z_{\mathrm{s}}\right)$. These comparisons show that the potential host galaxy contribution to $\mathrm{DM}_{\mathrm{exc}}$ increases the likelihood of an FRB to have a $\mathrm{DM}_{\mathrm{exc}}$ in excess of that predicted by conventional analysis. This excess may be particularly large for sources at low redshifts. In some cases (see e.g. subplots (a) and (c), which trace YPSR distributions in spiral galaxies and elliptical distributions in elliptical galaxies, respectively), the influence of the host diminishes significantly by $z_{\mathrm{s}}=3$, signified by the convergence of the $P\left(\mathrm{DM}_{\mathrm{exc}}\right)$ curves with their IGM counterparts. In other cases (see e.g. subplots (c) and (f), where FRBs are confined to galactic regions of high electron density), the host's potential influence is still significant at $z_{\mathrm{s}}=3$.

Differences between $P\left(\mathrm{DM}_{\mathrm{exc}} \mid z_{\mathrm{s}}\right)$ profiles are also apparent between different progenitor models, particularly for sources

6 FRB 110523's observed magnetisation and scattering properties may also indicate a similar environment (Masui et al. 2015). with low redshifts. The $\mathrm{DM}_{\mathrm{exc}}$ number density profiles acquired for large samples of redshift-constrained FRBs could therefore prove valuable tools for FRB progenitor analysis (see Sect. 4.3). We note that each of our models considers a single progenitor scenario. In reality, a combination of these models may be more representative of the true FRB population.

\section{2. $P(z / \mathrm{DM})$ methodology and results}

Applying Bayes' theorem to Eq. (16):

$P\left(z_{\mathrm{s}} \mid \mathrm{DM}_{\mathrm{exc}}\right)=\frac{P\left(\mathrm{DM}_{\mathrm{exc}} \mid z_{\mathrm{s}}\right) P\left(z_{\mathrm{s}}\right)}{P\left(\mathrm{DM}_{\mathrm{exc}}\right)}$,

where the PDF $P\left(z_{\mathrm{s}} \mid \mathrm{DM}_{\mathrm{exc}}\right)$ describes the likelihood that an FRB with a measured time delay $\Delta t_{\text {obs,exc }}$ between observing frequencies $\left(v_{1, \mathrm{obs}}\right.$ and $\left.v_{2, \mathrm{obs}}\right)$ on Earth due to a DM excess $\mathrm{DM}_{\mathrm{exc}}$ will have a source redshift $z_{\mathrm{s}}$ according to the IGM and host models comprising $P\left(\mathrm{DM}_{\mathrm{exc}} \mid z_{\mathrm{s}}\right)$. For practical application the model should be normalised to $\int P\left(z_{\mathrm{s}} \mid \mathrm{DM}_{\mathrm{exc}}\right) \mathrm{d} z=1$. In Eq. (17), $P\left(\mathrm{DM}_{\mathrm{exc}}\right)$ is the probability of observing a DM excess and $P\left(z_{\mathrm{s}}\right)$ is the assumed prior FRB redshift distribution.

Figure 5 shows projections of $P\left(z_{\mathrm{s}} \mid \mathrm{DM}_{\mathrm{exc}}\right)$ for FRBs of different $\mathrm{DM}_{\mathrm{exc}}$ values assuming a progenitor population following a YPSR spacial distribution in spiral galaxies. The left subplot compares projections against $P\left(z_{\mathrm{s}} \mid \mathrm{DM}_{\mathrm{IGM}}\right)$, illustrating the potential influence of the host galaxy on probable redshifts of an FRB. The comparisons illustrate the diminishing influence of the host with increasing $\mathrm{DM}_{\mathrm{exc}}$ owing to the increased likelihood of a high-redshift source. This result has implications for constraining redshifts using their DMs alone. Using such techniques, FRBs with larger $\mathrm{DM}_{\text {exc }}$ values are more accurately constrained. We note that the underlying progenitors of FRBs can significantly alter the redshift range available to an FRB (see Sect. 4.3), so the most accurate constraints result from use of realistic host and IGM models.

The assumed prior FRB redshift distribution $P\left(z_{\mathrm{s}}\right)$ may also influence the potential redshift ranges available to FRBs. The uniform prior $(\mathrm{U}(0,6))$ considered across the generated redshift range in Fig. 5 (left) assumes that FRBs are equally distributed with redshift. Redshift distributions of FRBs rely on their progenitors, so this may not be the case. Zhou et al. (2014) previously considered the redshift distribution of FRBs to follow an Erlang distribution fit, i.e.

$P\left(z_{\mathrm{s}} ; k, \gamma\right)=\gamma^{k} z_{\mathrm{s}}^{k-1} e^{-\gamma z_{\mathrm{s}}} / \Gamma(k)$,

where $k=2, \gamma=1$ which has been associated with GRBs by Shao et al. 2011 and accounts for a dearth of observed events in the nearby Universe (presumably owing to the relative scarcity of galaxies), and the decreased likelihood of detection of distant events by sensitivity-limited instruments. Figure 5 (right) compares $P\left(z_{\mathrm{s}} \mid \mathrm{DM}_{\mathrm{exc}}\right)$ projections assuming uniform and Erlang priors for an FRB of $\mathrm{DM}_{\mathrm{exc}}$ equal to that of FRB 121102. The Erlang prior suppresses the likelihood of its source originating at low redshifts. Both distributions are still consistent with spectroscopic measurements of FRB 121102's host galaxy.

\section{Discussion and conclusions}

In previous sections we have reviewed the contributing components to observed FRB DMs, their redshift dependences, and have combined physically motivated PDFs for these components. Excess electron models resulting from such techniques allow 

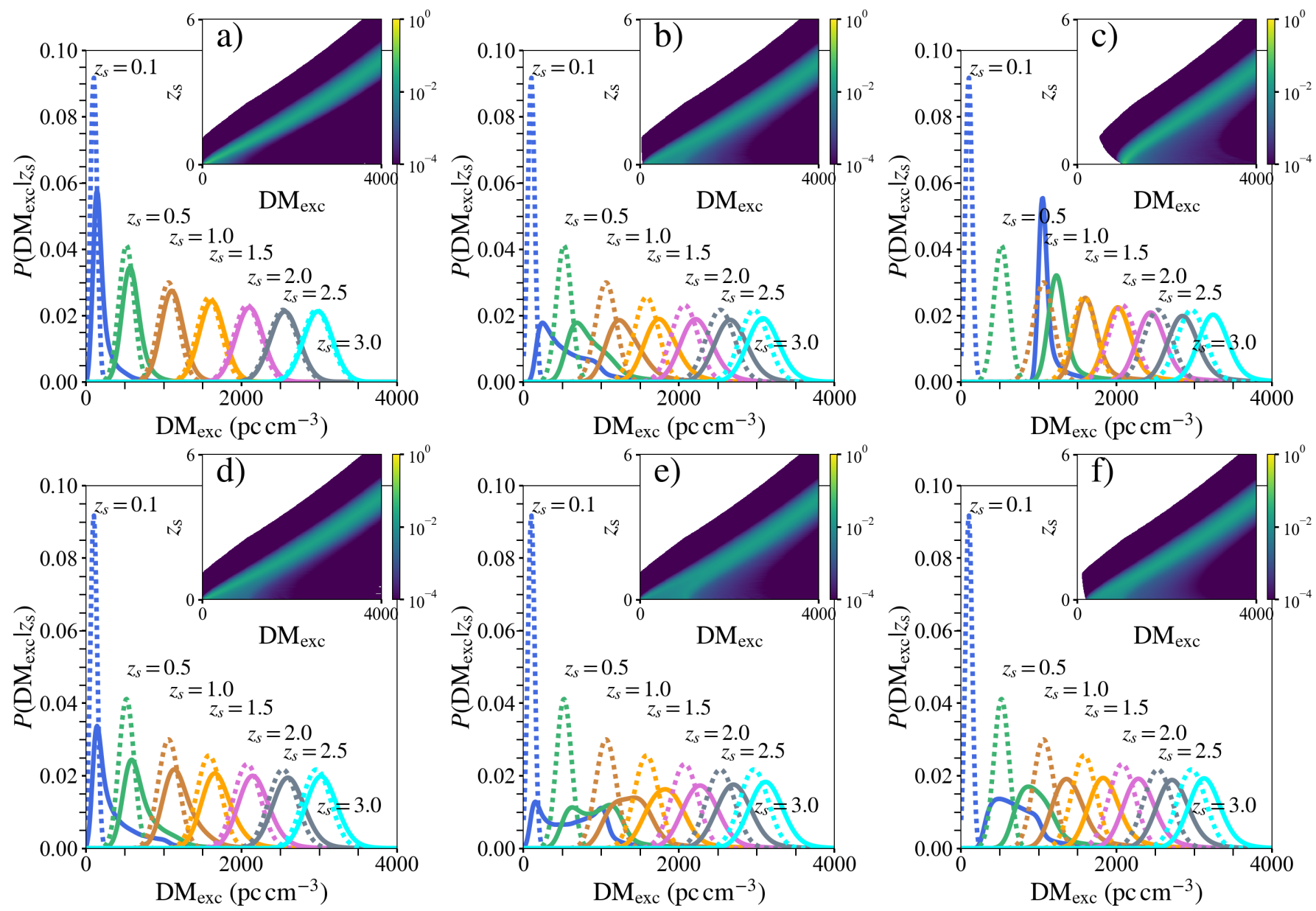

Fig. 4. Excess electron models $P\left(\mathrm{DM}_{\mathrm{exc}} z_{\mathrm{s}}\right)$ illustrating the relationship between observed excess dispersion measure DM $\mathrm{exc}_{\text {and source redshift }}$ $z_{\mathrm{s}}$ for simulated FRB populations. Subplots consider progenitor scenarios following: (a) YPSR distributions in spiral galaxies, homogeneous distributions within galactocentric spheres of radius; $(b) H=0.2 \mathrm{kpc} ;(c) H=0.004 \mathrm{kpc}$ in spiral galaxies; elliptical distributions confined to regions $(d) 1 ;(e) 0.1$ times the effective radius $\left(R_{\mathrm{e}}=3.2 \mathrm{kpc}\right)$ of elliptical galaxies; and $(f)$ homogeneous distributions within galactocentric spheres of radius $H=0.1 \mathrm{kpc}$ in elliptical galaxies. Projections of each model for different $z_{\mathrm{s}}$ (solid lines) are compared with $P\left(\mathrm{DM}_{\mathrm{IGM}} \mid z_{\mathrm{s}}\right)\left(\mathrm{dotted}_{\mathrm{f}}\right.$ lines) to illustrate the influence of the host contribution. The two-dimensional PDFs themselves $\left(z_{\mathrm{s}}\right.$ against $\mathrm{DM}_{\mathrm{exc}}$ with colour indicating relative likelihood) are inset into each subplot. Models are normalised to ensure $\int P\left(\mathrm{DM}_{\mathrm{exc}} \mid z_{\mathrm{s}}\right) \mathrm{dDM}=1$.

more accurate constraints to be placed on unlocalised FRB redshifts than arise from standard practice. This is beneficial as an FRB's observed DM will always be well-defined regardless of whether or not a spectroscopic redshift may be obtained. The threefold purposes of this work are as follows. Firstly, we seek to improve FRB DM-redshift analysis at $z_{\mathrm{s}}>2$, by considering the redshift-evolving mean in $\mathrm{DM}_{\mathrm{IGM}}$ and its associated variance due to galactic haloes along sightlines, the contribution of the host galaxy to $\mathrm{DM}_{\mathrm{exc}}$ assuming different progenitor scenarios, and the effects of underlying prior FRB redshift distributions on their potential redshift ranges. Furthermore, we aim to illustrate the differences that physically-motivated models have on FRB DM-redshift probability distributions. Secondly, we place constraints on the redshifts of unlocalised FRBs using their DMs given the above considerations. Finally, we identify potential uses for DM-redshift analysis in future FRB research.

\subsection{Dispersion measure component analysis}

For all FRB progenitor scenarios we consider, our $P\left(\mathrm{DM}_{\text {host }} \mid z_{\mathrm{s}}=\right.$ 0 ) curves indicate potentially significant rest-frame dispersion $\left(\mathrm{DM}_{\text {host }}>400 \mathrm{pc} \mathrm{cm}^{-3}\right.$, and in some cases $\left.>700 \mathrm{pc} \mathrm{cm}^{-3}\right)$ by the host galaxy (see Fig. 3). This result is consistent with previous spiral galaxy dispersion studies (e.g. Zhou et al. 2014; Xu \& Han 2015). Our simulations also predict potentially nonnegligible dispersion by elliptical galaxies, which conflicts with past literature (e.g. Xu \& Han 2015). This discrepancy may be a result of different modelling methods. Xu \& Han (2015) scale a Milky Way-sized elliptical galaxy (constructed by neglecting NE2001's thin disc and spiral arm components) using $\mathrm{H}_{\alpha}$ to trace ionised electrons, whereas we use a $\beta$ model and confine sources to areas within the effective radius of the galaxy. We also note that our elliptical models are constructed to approach the local IGM electron density at their respective virial radii. However, excess matter past this point could in principle contribute to $\mathrm{DM}_{\text {host }}$. Therefore future work could utilise an improved elliptical model obtained by theoretically deriving the electron number density profile of Eq. (8) and deducing the true radius at which it equals the cosmic mean.

Figure 4 indicates that the relative host galaxy contribution to $\mathrm{DM}_{\mathrm{exc}}$ is most influential at low $z_{\mathrm{s}}$ and diminishes as $z_{\mathrm{s}}$ increases. This effect, due to cosmological time dilation and host galaxy recession (Ioka 2003), is independent of progenitor/host model and has previously been noted to inadequately suppress host 

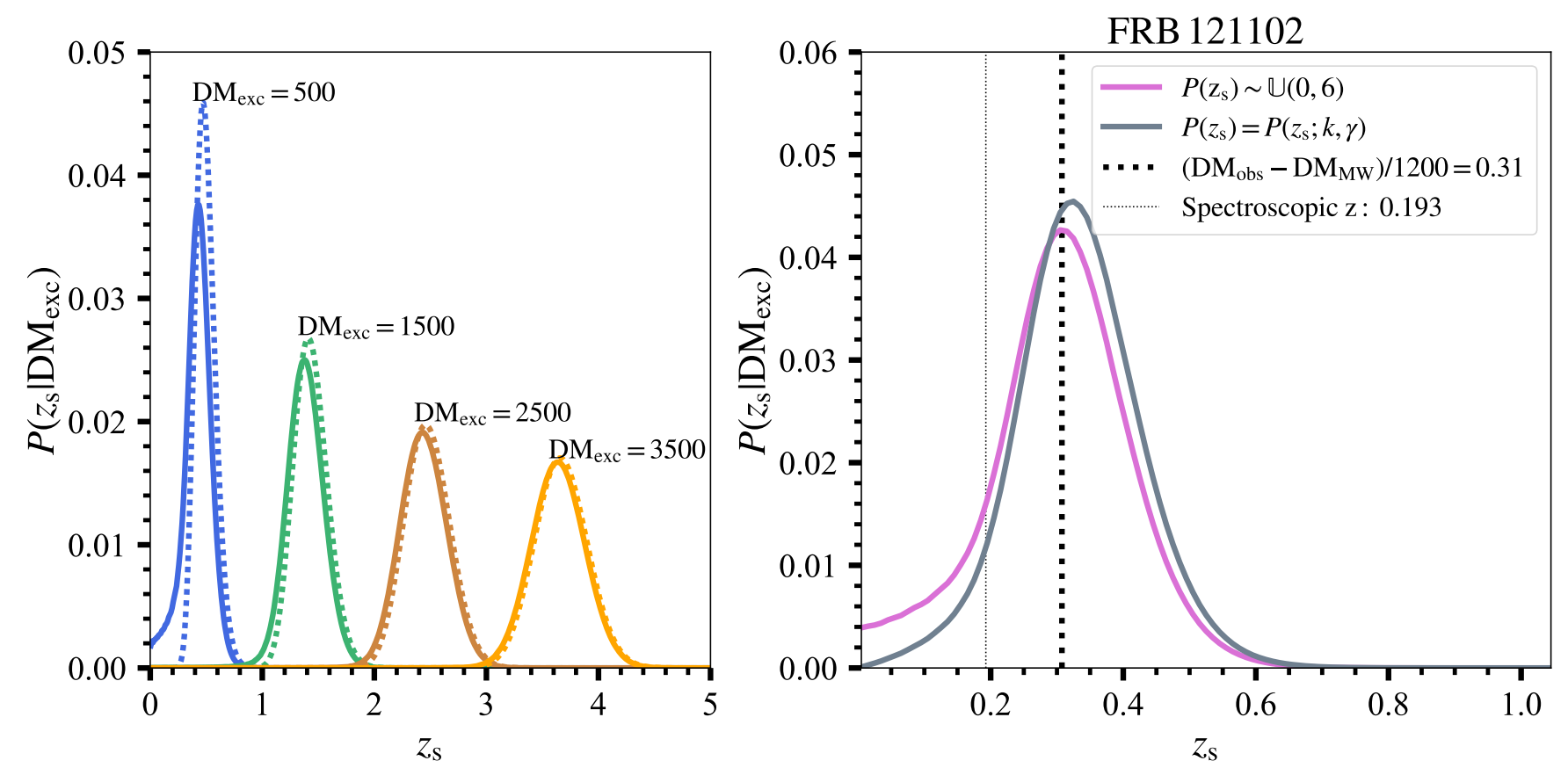

Fig. 5. $P\left(z_{\mathrm{s}} \mid \mathrm{DM}_{\mathrm{exc}}\right)$ projections after application of Bayes' theorem, illustrating potential source redshift $z_{\mathrm{s}}$ ranges for FRBs with observed excess electron components $\mathrm{DM}_{\mathrm{exc}}$. The model chosen assumes FRB progenitors to follow YPSR distributions in spiral galaxies. Left subplot: DM projections (solid lines) against $P\left(z_{\mathrm{s}} \mid \mathrm{DM}_{\mathrm{IGM}}\right)$ (dashed lines), illustrating the influence of the host contribution. A uniform (U(0,6) prior redshift distribution is assumed. Right subplot: projections for an FRB of $\mathrm{DM}_{\mathrm{exc}}$ equal to that of FRB 121102. Uniform (pink) and Erlang $\left(P\left(z_{\mathrm{s}}\right)=P\left(z_{\mathrm{s}} ; k, \gamma\right)\right)$ prior redshift distributions are compared. Vertical lines indicating FRB 121102's redshift as predicted conventionally (dash-dotted) and as observed spectroscopically (dashed) are included. Models are normalised to ensure $\int P\left(z_{\mathrm{s}} \mid \mathrm{DM}\right) \mathrm{d} z=1$.

galaxy contributions $>700 \mathrm{pc} \mathrm{cm}^{-3}$ for FRBs of $z_{\mathrm{s}}<2$ (Zhou et al. 2014; Macquart et al. 2015). Our models show that in some cases progenitor/host scenarios may significantly influence an FRB's potential $\mathrm{DM}_{\mathrm{exc}}$ range out to $z_{\mathrm{s}}>3$ (e.g. when sources are confined to regions of high electron density).

Our chosen models may not accurately represent the origins of individual FRBs. FRB 121102, the only spectroscopically constrained source to date, originates in a dwarf galaxy (Tendulkar et al. 2017), which is a scenario we have not considered. During the analysis we hold our galaxy masses fixed, which could be improved by considering realistic host galaxy mass distributions (see e.g. Luo et al. 2018). As our assumed masses lie close to the top of the mass range, such considerations may further suppress the overall host contribution to $P\left(\mathrm{DM}_{\mathrm{exc}} \mid z_{\mathrm{s}}\right)$ relative to that of the IGM. Future DM-redshift analysis would also ideally incorporate a $\mathrm{DM}_{\text {local }}$ component. Finally, we note that we idealistically assume perfectly removed $\mathrm{DM}_{\mathrm{MW}}$ components in this paper. Uncertainties in Milky Way disc and halo electron density distribution models must be considered for the most accurate DM-redshift analysis of FRBs.

\subsection{Redshift constraints for unlocalised FRBs}

Table B.1 presents revised source redshifts for all catalogued FRBs to date, constrained to bounds drawn from their $95 \%$ confidence intervals. The table includes progenitor scenarios chosen to highlight the impact of the host/progenitor on an FRB's potential redshift range. Our chosen progenitor scenarios are as follows:

a) Distributions neglecting a $\mathrm{DM}_{\text {host }}$ component. For FRBs of $z_{\mathrm{s}}<2$, these distributions essentially recover conventionally calculated redshifts. b) Sources tracing young pulsar populations in Milky Way-like spiral galaxies.

c) Sources confined to galactocentric spheres of radius $H=$ $0.2 \mathrm{kpc}$ in Milky Way-like spiral galaxies.

d) Sources tracing the gas profiles of $5 \times 10^{10} M_{\odot}$ elliptical galaxies, confined to areas within their $R_{\mathrm{e}}=3.2 \mathrm{kpc}$ effective radii.

e) Sources confined to galactocentric spheres of radius $H=$ $0.1 \mathrm{kpc}$ in $5 \times 10^{10} M_{\odot}$ elliptical galaxies.

In all cases a $U(0,6)$ prior FRB redshift distribution has been assumed. The true cosmological FRB redshift distribution depends on FRB phenomenology.

We provide our models and a Jupyter notebook with this work so that our results may be reproduced, and so that others may obtain redshifts for FRBs with our models or substitute their own $P\left(\mathrm{DM}_{\mathrm{IGM}} \mid z_{\mathrm{s}}\right), P\left(\mathrm{DM}_{\text {host }} \mid z_{\mathrm{s}}=z_{0}\right)$, and $P\left(z_{\mathrm{s}}\right)$ distributions. We propose that such techniques be considered for general use during future FRB analysis to better constrain the redshifts of unlocalised FRBs.

\subsection{Potential uses for DM-redshift analysis}

In this section, we identify potential implications of the DMredshift relationship and uses for the framework presented in this paper.

Progenitor distinction. Figure 4 illustrates the potentially significant impact of FRB host galaxies on their possible $\mathrm{DM}_{\mathrm{exc}}$ values. Therefore observed $\mathrm{DM}_{\mathrm{exc}}$ distributions for FRBs constrained to $z_{\mathrm{s}}$ ranges may be useful for ruling out progenitor scenarios. We illustrate the approach for the set of models described in Sect. 4.2. We consecutively draw $\mathrm{DM}_{\text {exc }}$ samples of increasing size from a model in the set held to be true and 

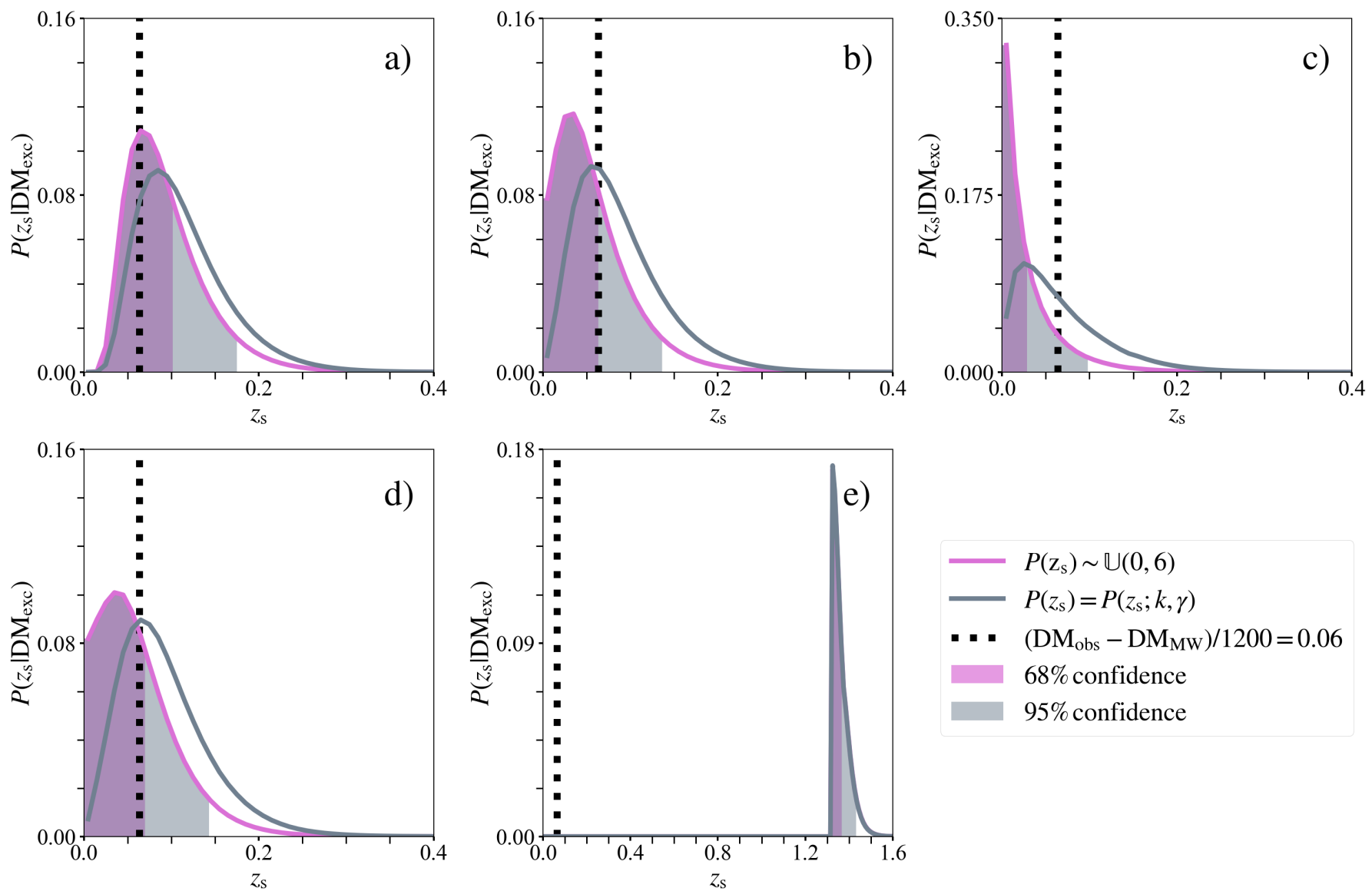

Fig. 6. $P\left(z_{\mathrm{s}} \mid \mathrm{DM}_{\mathrm{exc}}\right)$ projections after application of Bayes' theorem for an FRB of $\mathrm{DM}_{\mathrm{exc}}$ equal to that of FRB 171020. Subplots consider progenitor scenarios following: $(a)$ distributions neglecting a host galaxy DM component, $(b)$ YPSR distributions in spiral galaxies, $(c)$ homogeneous distributions within galactocentric spheres of radius $H=0.2 \mathrm{kpc}$ in spiral galaxies, $(d)$ elliptical distributions confined to the effective radii $\left(R_{\mathrm{e}}=3.2 \mathrm{kpc}\right)$ of elliptical galaxies, and $(e)$ homogeneous distributions within galactocentric spheres of radius $H=0.1 \mathrm{kpc}$ in elliptical galaxies. Uniform (pink lines) and Erlang (grey lines) prior redshift distributions are compared. Vertical lines indicate the conventionally calculated redshift of the FRB. Shaded regions indicate bounds drawn from 68 to $95 \%$ confidence intervals. Models are normalised to unity.

perform 10000 Bayesian model selection tests against the other models. We define the threshold for unambiguous distinction of a "true" model from all others to be when $99.7 \%$ of tests result in "decisive" evidence (i.e. $\log _{10}(k)>2$, where $k$ is the Bayes factor) in favour of the true model. We find that unambiguous distinction of any true model from the remainder of the set requires between 200-300, 300-400, or 700-800 FRBs with accurate $\mathrm{DM}_{\mathrm{exc}}$ values and source redshifts between $0.45<z_{\mathrm{s}}<$ $0.65,0.95<z_{\mathrm{s}}<1.05$, or $1.45<z_{\mathrm{s}}<1.65$, respectively. To distinguish between models of sources tracing stellar distributions (e.g. model b) and sources confined to regions of high electron density (e.g. models c, e), fewer than 50 FRBs are required for any of these redshift ranges.

Host galaxy rejection. An observed FRB of unconstrained $z_{\mathrm{s}}$ always has a well-defined $\mathrm{DM}_{\mathrm{obs}}$. After subtracting an accurate $\mathrm{DM}_{\mathrm{MW}}$, progenitor model and prior redshift distributiondependent $P\left(z_{\mathrm{s}} \mid \mathrm{DM}_{\mathrm{exc}}\right)$ curves may be used to constrain $z_{\mathrm{s}}$ to a statistical significance. Galaxies within the FRB localisation region, but outside the selected redshift range may then be discounted as potential hosts. We implement this technique for the low-DM ${ }_{\text {obs }}\left(=114 \mathrm{pc} \mathrm{cm}^{-3}\right)$ FRB 171020, motivated by the work of Mahony et al. (2018).

Figure 6 shows $P\left(z_{\mathrm{s}} \mid \mathrm{DM}_{\mathrm{exc}}\right)$ projections for the burst assuming the progenitor scenarios in Sect. 4.2. Table B.1 contains numerical constraints for their $95 \%$ confidence regions. The potential available $z_{\mathrm{s}}$ range is shown to be significantly influenced by both progenitor model and $P\left(z_{\mathrm{s}}\right)$. Models (a)-(d) are broadly consistent with Mahony et al. (2018) calculations ${ }^{7}$ but with larger upper limits $\left(0.098<z_{\mathrm{s}, \max }<0.143\right)$. Model e differs significantly from these ranges $\left(1.325<z_{\mathrm{s}}<1.432\right)$. This result may be understood by considering the large $z_{\mathrm{s}}$ necessary for relativistic effects to suppress sufficiently the large $\mathrm{DM}_{\text {host }}$ acquired from an electron-dense environment. A search of the NASA Extragalactic Database (NED) within the localisation region of the FRB using our revised $z_{\mathrm{s}}$ ranges yields 2148 objects, 13 of which have spectroscopically constrained redshifts. Of these, 11 fall outside our ranges for models (a) $-(d)$. The remaining two galaxies are discussed in Mahony et al. (2018). If FRB 171020 were to have originated from a model (e)-like progenitor, these two galaxies could also be discounted as potential hosts.

Milky Way uncertainty mitigation. By observing small areas of sky, surveys may mitigate uncertainty as a consequence of $\mathrm{DM}_{\mathrm{MW}}$ sightline variations. Over a small enough area, the Milky Way contribution to $\mathrm{DM}_{\mathrm{obs}}$ could be considered uniform, resulting in a constant systematic offset in the $\mathrm{DM}_{\mathrm{exc}}$ distribution for all FRBs observed in any given direction. Current and future FRB searches capable of piggybacking deep extragalactic

\footnotetext{
$\overline{7 z_{\mathrm{s}}<0.08, \text { assuming } \mathrm{DM}_{\text {host }}}=45 \mathrm{pc} \mathrm{cm}^{-3}$ and a $z_{\mathrm{s}} \simeq \mathrm{DM}_{\mathrm{IGM}} / 1000$ relationship.
} 
surveys (e.g. V-FASTR, MEETRAP, LOFT-e; Wayth et al. 2011; Burke-Spolaor et al. 2016; Stappers 2016; Walker et al. 2018) could potentially provide such sets of FRBs. Assuming FRBs have no preferential latitude dependence (Bhandari et al. 2018), this approach would yield similar numbers of events in any pointing direction.

$D M_{\mathrm{MW}}$ analysis. By considering the above approach, sufficient numbers of pointings by deep extragalactic FRB searches over large sky areas collecting sufficiently large numbers of FRBs would allow the disentanglement of $\mathrm{DM}_{\mathrm{exc}}$ and $\mathrm{DM}_{\mathrm{MW}}$. Assuming the true underlying $P\left(\mathrm{DM}_{\mathrm{exc}} \mid z_{s}\right)$ to be directionindependent, the $\mathrm{DM}_{\mathrm{obs}}$ distribution for FRBs of given $z_{\mathrm{s}}$ observed in different directions could be compared and searched for systematic offsets.

We demonstrate this technique by analysing how well an underlying $\mathrm{DM}_{\mathrm{MW}}$ distribution may be recovered for a simulated FRB population. To do so, we generated an all-sky FRB population, with $\mathrm{DM}_{\mathrm{MW}}$ and $\mathrm{DM}_{\mathrm{exc}}$ components drawn from the NE2001 model and a generic normal distribution, respectively. The latter distribution was chosen for simplicity, as the true distribution is irrelevant in this situation (see below). We then divided the sky into regions small enough to have constant $\mathrm{DM}_{\mathrm{MW}}$ using a HEALPix tessellation; we experimented with $N$ Side $=4,8$, and 16. Assuming the $P\left(\mathrm{DM}_{\mathrm{obs}} \mid z_{\mathrm{s}}\right)$ distribution to be the same in all directions except for position-dependent offset due to the $\mathrm{DM}_{\mathrm{MW}}$ contribution, we can recover the relative distribution of this Milky Way component across the sky by calculating the average DM within each tile. An intrinsic uncertainty in the overall zero point of $\mathrm{DM}_{\mathrm{MW}}$ exists owing to the unknown mean $\mathrm{DM}$ of $P\left(\mathrm{DM}_{\mathrm{exc}} \mid z_{\mathrm{s}}\right)$. However, the relative reconstruction of $\mathrm{DM}_{\mathrm{MW}}$ is independent of the extragalactic probability distribution, thus justifying our earlier choice of a simple normal distribution. Our simulations show that we can recover $\mathrm{DM}_{\mathrm{MW}}$ to $\sim 37(\mathrm{nFRB} / \text { tile })^{-1 / 2} \mathrm{pc} \mathrm{cm}^{-} 3$ for any sky-tile tile $\lesssim 200$ sq. deg., provided that it is far enough from the Galactic plane (i.e. $\sim 5^{\circ}$ ) for the DM gradient within it to be fairly small.

The same reconstruction technique can also be applied to flux-limited surveys (e.g. CRAFT, CHIME/FRB; Macquart et al. 2010; CHIME/FRB Collaboration 2018) since $P\left(\mathrm{DM}_{\text {exc }} \mid F>\right.$ $\left.F_{\text {lim }}\right)$ is also position-independent. For a representative all-sky survey containing $10000 \mathrm{FRBs}$, it would be possible to reconstruct the sky $\mathrm{DM}_{\mathrm{MW}}$ at a precision $\sim 4.6 \mathrm{pc} \mathrm{cm}^{-3}$, i.e. $\sim 10 \%$ relative uncertainty, at a $215 \mathrm{sq}$. deg. resolution. Interestingly, it would then be possible to remove the MW contribution from all FRBs and combine these in order to obtain a proper $P\left(\mathrm{DM}_{\text {exc }} \mid F>F_{\text {lim }}\right)$ that is accurate at the $\sim 2 \%$ level as far as recovering the second moment is concerned.

As cosmological analysis of future redshift-constrained FRB populations relies on precise removal of $\mathrm{DM}_{\mathrm{MW}}$, this application is a potentially crucial case for obtaining large numbers of FRBs with a limited localisation accuracy.

$D M_{\text {IGM }}-D M_{\text {host }}$ disentanglement. As $\operatorname{DM}_{\mathrm{IGM}}\left(z_{\mathrm{s}}\right)$ is a function integrated over redshift while $\operatorname{DM}_{\text {host }}\left(z_{\mathrm{s}}\right)$ is evaluated at a specific source redshift, the two components may theoretically be disentangled using redshift-constrained FRBs. Techniques adapted by analogy to frequency-dependent component separation of Galactic foreground and cosmic microwave background (CMB) emission (see, e.g. Planck Collaboration IX 2016) may be of use. However, even though the redshift dependence of these components is well known, their true underlying PDFs is not readily extractable. Thus component separation may require forward modelling using realistic template $P\left(\mathrm{DM}_{\mathrm{IGM}} \mid z_{\mathrm{s}}\right)$ and $P\left(\mathrm{DM}_{\text {host }} \mid z_{\mathrm{s}}\right)$ distributions or a decomposition into basis functions such as a Gaussian mixture.

\subsection{Concluding remarks}

We present in this paper a statistical framework for the exploration of the FRB DM-redshift relationship, which provides the basis for several analyses. Firstly, it facilitates the assessment of host galaxy contributions to FRB DMs using physically motivated models. We find that all of our host models may potentially contribute rest-frame $\mathrm{DM}_{\text {host }}$ values $>400 \mathrm{pc} \mathrm{cm}^{-3}$. By applying relativistic effects in an expanding Universe, we demonstrate the diminishing influence of $\mathrm{DM}_{\text {host }}$ with increasing redshift. But for extreme progenitor scenarios, in which FRBs originate in electron-dense regions (e.g. near to galactic centres), the $\mathrm{DM}_{\text {host }}$ component may still significantly influence $P\left(\mathrm{DM}_{\mathrm{exc}} \mid z_{\mathrm{s}}\right)$ profiles out to $z_{\mathrm{s}}>3$. We place limits on the number of semi-redshiftconstrained FRBs required to distinguish between our models, finding that $<50$ events may be required to identify galactocentric from stellar progenitor populations. Secondly, the framework may be used for constraining the redshifts of unlocalised FRBs more rigorously than by using conventional techniques. By consulting $P\left(z_{\mathrm{s}} \mid \mathrm{DM}\right)$ probability distributions generated using physically motivated progenitor models, we provide constraints for all FRBs catalogued to date. We use these constraints to reject host galaxy candidates within the localisation region of

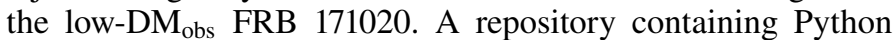
code and examples of the DM-redshift analysis demonstrated in this work is provided online ${ }^{8}$. Finally, such techniques may aid $\mathrm{DM}_{\mathrm{MW}}$ uncertainty mitigation and $\mathrm{DM}_{\mathrm{obs}}$ component disentanglement. Assuming a direction-independent $P\left(\mathrm{DM}_{\mathrm{exc}} \mid z_{\mathrm{s}}\right)$, the $\mathrm{DM}_{\mathrm{MW}}$ contribution to $\mathrm{DM}_{\mathrm{obs}}$ could be identified with a search for sightline-variable offsets in histogrammed $\mathrm{DM}_{\mathrm{exc}}$ distributions obtained by flux-limited surveys (e.g. CHIME/FRB). Surveys yielding many FRBs within small enough redshift ranges could potentially put FRBs without spectroscopic redshift measurements to use with this technique. It may also be possible to separate $\mathrm{DM}_{\mathrm{IGM}}$ and $\mathrm{DM}_{\text {host }}$ using their respective redshift dependences.

Acknowledgements. We thank Francesco Pace for vital conversation and instruction during this project, Matthew McQuinn for providing useful IGM comparison models, and Joseph Callingham, Jason Hessels, Cherry Ng and the anonymous referee for their suggestions for improving this paper. C.R.H.W. acknowledges support from a UK Science and Technology Facilities Council studentship. R.P.B. acknowledges support from the ERC under the European Union's Horizon 2020 research and innovation programme (grant agreement no. 715051; Spiders). Y.Z.M. is supported by the National Research Foundation of South Africa with Grant no. 105925 and no. 104800.

\section{References}

Bannister, K. W., Shannon, R. M., Macquart, J.-P., et al. 2017, ApJ, 841, L12

Bassa, C. G., Tendulkar, S. P., Adams, E. A. K., et al. 2017, ApJ, 843, L8

Bhandari, S., Keane, E. F., Barr, E. D., et al. 2018, MNRAS, 475, 1427

Brown, B. A., \& Bregman, J. N. 2001, ApJ, 547, 154

Burke-Spolaor, S., Trott, C. M., Brisken, W. F., et al. 2016, ApJ, 826, 223

Caleb, M., Flynn, C., Bailes, M., et al. 2017, MNRAS, 468, 3746

Chatterjee, S., Law, C. J., Wharton, R. S., et al. 2017, Nature, 541, 58

CHIME/FRB Collaboration (Amiri, M., et al.) 2018, ApJ, 863, 48

Cordes, J. M., \& Lazio, T. J. W. 2002, ArXiv e-prints [astro-ph/0207156]

Cordes, J. M., \& Wasserman, I. 2016, MNRAS, 457, 232

de Graaff, A., Cai, Y.-C., Heymans, C., \& Peacock, J. A. 2019, A\&A, 624, A48

Dodelson, S. 2003, Modern Cosmology (Cambridge, US: Academic Press)

8 https://doi.org/10.5281/zenodo. 1209920 
C. R. H. Walker et al.: Constraining the redshifts of unlocalised fast radio bursts

Falcke, H., \& Rezzolla, L. 2014, A\&A, 562, A137

Gómez, G. C., Benjamin, R. A., \& Cox, D. P. 2001, AJ, 122, 908

Ioka, K. 2003, ApJ, 598, L79

Kalberla, P. M. W., \& Dedes, L. 2008, A\&A, 487, 951

Keane, E. F. 2016, MNRAS, 459, 1360

Keane, E. F. 2018, Nat. Astron., 2, 865

Lorimer, D. R. 2013, IAU Symp., 291, 237

Lorimer, D. R., Bailes, M., McLaughlin, M. A., Narkevic, D. J., \& Crawford, F. 2007, Science, 318, 777

Luo, R., Lee, K., Lorimer, D. R., \& Zhang, B. 2018, MNRAS, 481, 2320

Ma, Y.-Z., \& Zhao, G.-B. 2014, Phys. Lett. B, 735, 402

Macquart, J.-P., Bailes, M., Bhat, N. D. R., et al. 2010, PASA, 27, 272

Macquart, J. P., Keane, E., Grainge, K., et al. 2015, Advancing Astrophysics with the Square Kilometre Array (Berlin: Springer), 55

Mahony, E. K., Ekers, R. D., Macquart, J.-P., et al. 2018, ApJ, 867, L10

Maíz-Apellániz, J. 2001, AJ, 121, 2737

Mamon, G. A., \& Łokas, E. L. 2005, MNRAS, 363, 705

Manchester, R. N., \& Taylor, J. H. 1972, Astrophys. Lett., 10, 67

Marcote, B., Paragi, Z., Hessels, J. W. T., et al. 2017, ApJ, 834, L8

Masui, K., Lin, H.-H., Sievers, J., et al. 2015, Nature, 528, 523

McQuinn, M. 2014, ApJ, 780, L33

Michilli, D., Seymour, A., Hessels, J. W. T., et al. 2018, Nature, 553, 182

Mo, H. J., \& White, S. D. M. 2002, MNRAS, 336, 112

Mottez, F., \& Zarka, P. 2014, A\&A, 569, A86

Ng, C., Vanderlinde, K., Paradise, A., et al. 2017, ArXiv e-prints, [arXiv:1702.04728]

Nicastro, F., Kaastra, J., Krongold, Y., et al. 2018, Nature, 558, 406

Pen, U.-L., \& Connor, L. 2015, ApJ, 807, 179

Petroff, E., Barr, E. D., Jameson, A., et al. 2016, PASA, 33, e045
Planck Collaboration IX. 2016, A\&A, 594, A9

Planck Collaboration VI. 2020, A\&A, in press, https://doi.org/10.1051/ Q004-6361/201833910

Romero, G. E., del Valle, M. V., \& Vieyro, F. L. 2016, Phys. Rev. D, 93 023001

Schnitzeler, D. H. F. M. 2012, MNRAS, 427, 664

Scoville, N., Aussel, H., Brusa, M., et al. 2007, ApJS, 172,

Shao, L., Dai, Z.-G., Fan, Y.-Z., et al. 2011, ApJ, 738, 19

Spitler, L. G., Cordes, J. M., Hessels, J. W. T., et al. 2014, ApJ, 790, 101

Spitler, L. G., Scholz, P., Hessels, J. W. T., et al. 2016, Nature, 531, 202

Stappers, B. 2016, in Proceedings of MeerKAT Science: On the Pathway to the SKA. 25-27 May, 2016 Stellenbosch, South Africa (MeerKAT2016), Online at https://pos.sissa.it/cgi-bin/reader/conf.cgi?confid=277, id. 10,10

Sun, X. H., \& Han, J. L. 2004, MNRAS, 350, 232

Tanimura, H., Hinshaw, G., McCarthy, I. G., et al. 2019, MNRAS, 483, 223

Tendulkar, S. P., Bassa, C. G., Cordes, J. M., et al. 2017, ApJ, 834, L7

Thornton, D., Stappers, B., Bailes, M., et al. 2013, Science, 341, 53

Totani, T. 2013, PASJ, 65, L12

Vieyro, F. L., Romero, G. E., Bosch-Ramon, V., Marcote, B., \& del Valle, M. V. 2017, A\&A, 602, A64

Walker, C. R. H., Breton, R. P., Harrison, P. A., et al. 2018, IAU Symp., 337, 422

Wayth, R. B., Brisken, W. F., Deller, A. T., et al. 2011, ApJ, 735, 97

Xu, J., \& Han, J. L. 2015, Res. Astron. Astrophys., 15, 1629

Yang, Y.-P., Luo, R., Li, Z., \& Zhang, B. 2017, ApJ, 839, L25

Yusifov, I., \& Küçük, I. 2004, A\&A, 422, 545

Zhang, B. 2018, ApJ, 867, L21

Zhou, B., Li, X., Wang, T., Fan, Y.-Z., \& Wei, D.-M. 2014, Phys. Rev. D, 89 107303 


\section{Appendix A: Variance in $\mathrm{DM}_{\mathrm{IGM}}$}

For our PDF of likelihood of a FRB of source redshift $z_{\mathrm{s}}$ to contribute $\mathrm{DM}_{\mathrm{IGM}}$ to the overall observed dispersion measure $\mathrm{DM}_{\mathrm{obs}}$, we assume a Gaussian

$P\left(\mathrm{DM}_{\mathrm{IGM}} \mid z_{\mathrm{s}}\right)=\frac{1}{\sqrt{2 \pi} \sigma_{\mathrm{DM}}} \exp \left(-\frac{\left(\mathrm{DM}-\mathrm{DM}_{\mathrm{IGM}}\left(z_{\mathrm{s}}\right)\right)^{2}}{2 \sigma_{\mathrm{DM}}^{2}}\right)$,

with mean

$\operatorname{DM}_{\mathrm{IGM}}\left(z_{\mathrm{s}}\right)=\int_{0}^{\chi\left(z_{\mathrm{s}}\right)} \mathrm{d} \chi \frac{n_{\mathrm{e}}(z)}{(1+z)^{2}}$

where the $n_{\mathrm{e}}(z)$ is the three-dimensional electron number density at redshift $z$, where the variance is

$\sigma_{\mathrm{DM}}^{2}\left(\mathrm{DM}, z_{\mathrm{s}}\right)=\int_{0}^{\chi_{\mathrm{s}}} \mathrm{d} \chi\left(1+z_{\mathrm{s}}\right)^{2} \bar{n}_{\mathrm{e}}^{2}(0) \int \frac{\mathrm{d}^{2} k_{\perp}}{(2 \pi)^{2}} P_{\mathrm{e}}\left(k_{\perp}, z_{\mathrm{s}}\right)$,

following McQuinn (2014). In this equation, $\chi$ is the comoving distance factoring out Universal expansion, $\mathrm{d} \chi=c \mathrm{~d} z / H(z)$, where $c$ is the speed of light and $H(z)$ is the Hubble factor at $z ; \bar{n}_{\mathrm{e}}(0)$ is the mean electron density at $z=0$; the matter power spectrum at $z$ for wavenumber $k$ is $P_{\mathrm{e}}(k, z)$; and $k_{\perp}$ is the perpendicular component (Dodelson 2003).

Following Eqs. (3) and (4), and Appendix (A.1)-(A.7) in Ma \& Zhao (2014), $n_{\mathrm{e}}(z)$ may be written as

$n_{\mathrm{e}}(z)=\frac{\chi_{\mathrm{e}} \rho_{\text {gas }}(z)}{\mu_{\mathrm{e}} m_{\mathrm{p}}}$,

where $\Omega_{\mathrm{b}}=0.048$ is the fractional baryon density (Planck Collaboration VI 2020), $m_{\mathrm{p}}$ is the proton mass, $\mu_{\mathrm{e}} \simeq 1.14$ is the $z)^{3}$ is the gas density at redshift $z$, where $\rho_{\text {cr }}=1.879 h^{2} \times$ $10^{-29} \mathrm{~g} \mathrm{~cm}^{-3}$ is the Universe's present critical density (Dodelson 2003), and mean mass per electron, $\rho_{\text {gas }}(z)=\rho_{\text {gas }}(z=0)(1+z)^{3}=\Omega_{\mathrm{b}} \rho_{\mathrm{cr}}(1+$

where $\chi_{\mathrm{e}}$ is the number ratio of ionised to total electrons, $Y_{\mathrm{p}} \simeq$ 0.24 is the helium mass fraction, and $N_{\mathrm{H}_{\mathrm{e}}}$ is the number of ionised electrons per helium atom. The quantity $N_{\mathrm{H}_{\mathrm{e}}}$ may range between $0-2$ therefore $\chi_{\mathrm{e}}$ may range from $0.86-1$. In this work we assume $\chi_{\mathrm{e}}=1$.

By changing variables,

$\mathrm{d} \chi=\frac{c \mathrm{~d} z}{H(z)}$,

Eq. (A.2) may be written more explicitly as

$\operatorname{DM}_{\mathrm{IGM}}\left(z_{\mathrm{s}}\right)=\left(\frac{\Omega_{\mathrm{b}} \chi_{\mathrm{e}} \rho_{\mathrm{cr}}}{m_{\mathrm{p}} \mu_{\mathrm{e}}}\right) \int_{0}^{z_{\mathrm{s}}} c \mathrm{~d} z \frac{(1+z)}{H(z)}$.

Substituting relevant quantities

$$
\begin{aligned}
\operatorname{DM}_{\mathrm{IGM}}\left(z_{\mathrm{s}}\right)= & 950.05\left[\mathrm{pc} \mathrm{cm}^{-3}\right]\left(\frac{\Omega_{\mathrm{b}}}{0.048}\right)\left(\frac{\chi_{\mathrm{e}}}{1.0}\right)\left(\frac{h}{0.67}\right) \\
& \times \int_{0}^{z_{\mathrm{s}}} \mathrm{d} z \frac{(1+z)}{E(z)},
\end{aligned}
$$

where $E(z)=\left[\Omega_{\mathrm{m}}(1+z)^{3}+\Omega_{\Lambda}\right]^{1 / 2}$ (Mo \& White 2002).

Likewise, following McQuinn (2014) the sightline variance in $\mathrm{DM}_{\mathrm{IGM}}$ is

$$
\begin{aligned}
\sigma_{\mathrm{DM}}^{2}(\mathrm{DM}) & =\int_{0}^{\chi_{\mathrm{s}}} \mathrm{d} \chi(1+z)^{2} \bar{n}_{\mathrm{e}}^{2}(0) \int \frac{\mathrm{d}^{2} k_{\perp}}{(2 \pi)^{2}} P_{\mathrm{e}}\left(k_{\perp}, z\right) \\
& =\frac{c}{H_{0}}\left[\frac{\chi_{\mathrm{e}} \Omega_{\mathrm{b}} \rho_{\mathrm{cr}}}{\mu_{\mathrm{e}} m_{\mathrm{p}}}\right]^{2} \int_{0}^{z_{\mathrm{s}}} \mathrm{d} z \frac{(1+z)^{2}}{E(z)} \int \mathrm{d} k k P_{\mathrm{e}}(k, z),
\end{aligned}
$$

where by Eq. (A.4)

$\chi_{\mathrm{e}}=\frac{1-Y_{\mathrm{p}}\left(1-N_{\mathrm{H}_{\mathrm{e}}} / 4\right)}{1-Y_{\mathrm{p}} / 2}$

$\bar{n}_{\mathrm{e}}(0)=\frac{\chi_{\mathrm{e}} \Omega_{\mathrm{b}} \rho_{\mathrm{cr}}}{\mu_{\mathrm{e}} m_{\mathrm{p}}}$. 


\section{Appendix B: Redshift constraints for existing FRBs}

Table B.1. DM-derived source redshifts $z_{\mathrm{s}}$ for catalogued FRBs (Col. 1) calculated via conventional techniques $\left(z_{\mathrm{s}} \sim \mathrm{DM}_{\mathrm{exc}} /\left(1200 \mathrm{pc} \mathrm{cm}^{-3}\right)\right.$ Petroff et al. 2016; Ioka 2003); (Col. 2), and from excess electron models described in Sect. 4.2 with bounds drawn from their $95 \%$ confidence intervals (Cols. 3-7).

\begin{tabular}{|c|c|c|c|c|c|c|}
\hline FRB & $z_{\mathrm{s}}($ conventional $)$ & $z_{\mathrm{s}}($ model a $)$ & $z_{\mathrm{s}}($ model b) & $z_{\mathrm{s}}($ model $\mathrm{c})$ & $z_{\mathrm{s}}($ model $\mathrm{d})$ & $\overline{z_{\mathrm{s}}(\operatorname{model~} \mathrm{e})}$ \\
\hline FRB180212 & 0.112 & $0.125_{-0.125}^{+0.126}$ & $0.085_{-0.085}^{+0.123}$ & $0.015_{-0.015}^{+0.130}$ & $0.085_{-0.085}^{+0.123}$ & $0.335_{-0.040}^{+0.129}$ \\
\hline FRB180131 & 0.515 & $0.585_{-0.570}^{+0.187}$ & $0.545_{-0.273}^{+1.370}$ & $0.445_{-0.396}^{+1.380}$ & $0.525_{-0.425}^{+1.370}$ & $0.285_{-0.261}^{+1.480}$ \\
\hline FRB180130 & 0.254 & $0.295_{-0.295}^{+0.150}$ & $0.255_{-0.186}^{+1.220}$ & $0.155_{-0.141}^{+1.270}$ & $0.245_{-0.210}^{+1.220}$ & $0.005_{-0.005}^{+0.199}$ \\
\hline FRB 180128.2 & 0.380 & $0.435_{-0.420}^{+0.171}$ & $0.395_{-0.251}^{+0.186}$ & $0.295_{-0.266}^{+0.1410}$ & $0.375_{-0.311}^{+0.2100}$ & $0.125_{-0.125}^{+0.005}$ \\
\hline FRB 180128.0 & 0.341 & $0.385_{-0.370}^{+0.460}$ & $0.345_{-0.229}^{+0.348}$ & $0.245_{-0.221}^{+0.266}$ & $0.335_{-0.281}^{+0.311}$ & $0.065_{-0.065}^{+0.1236}$ \\
\hline FRB180119 & 0.306 & $0.355_{-0.355}^{+0.155}$ & $0.305_{-0.230}^{-0.229}$ & $0.205_{-0.186}^{-0.221}$ & $0.295_{-0.248}^{+0.281}$ & $0.005_{-0.005}^{-0.065}$ \\
\hline FRB 180110 & 0.565 & $0.635_{-0.610}^{+0.355}$ & $0.605_{-0276}^{+0.2300}$ & $\begin{array}{r}-0.186 \\
0.495_{-0.439}^{+1.430}\end{array}$ & $0.575_{-0.457}^{+0.248}$ & $\begin{array}{r}-0.005 \\
0.335_{-0305}^{+1.510}\end{array}$ \\
\hline FRB171216 & 0.138 & $0.155^{-0.0132}$ & $0.115^{-0.2128}$ & $0.035^{-0.4340}$ & $0.115^{-0.457}$ & $\begin{array}{r}0.095_{-0.158}^{-0.305} \\
\end{array}$ \\
\hline FRB171213 & 0.102 & $0.115^{-0.155}$ & $0.075^{-0.1121}$ & $0.005^{-0.03130}$ & $0.085^{-0.1112}$ & $0.435_{-0.040}^{+0.040}$ \\
\hline FRB180817.J1533+42 & 0.816 & $0.915^{-0.227}$ & $0.875^{-0.0 .287}$ & $0.775^{-0.005}+.560$ & $0.845^{-0.085}+.540$ & $\begin{array}{r}-0.040 \\
0.625_{-0.562}^{+1.630}\end{array}$ \\
\hline FRB 180814.J1554+74 & 0.164 & $0.185^{-0.880}+137$ & $0.145^{-0.315}$ & $0.055^{-0.664}+150$ & $0.145^{+1.1 .170}$ & $0.005^{+0.562}$ \\
\hline FRB180814.J0422+73 & 0.085 & $\begin{array}{l}-0.185 \\
5^{+0.119}\end{array}$ & $\begin{array}{r}-0.139 \\
5^{+0.118}\end{array}$ & $\begin{array}{l}-0.055 \\
05^{+0.113}\end{array}$ & $0.065^{+0.110}$ & $\begin{array}{l}0.00 J_{-0.005}^{0.123} \\
0.675^{+0.123}\end{array}$ \\
\hline FRB180812 J0112+80 & 0.005 & $\begin{array}{l}0.07 J_{-0.095} \\
0675+0.200\end{array}$ & $\begin{array}{l}-0.055 \\
5+0.368\end{array}$ & $\begin{array}{l}-0.005 \\
25+1.450\end{array}$ & $0.00-0.065$ & $\begin{array}{l}.07-0.010 \\
375+1.530\end{array}$ \\
\hline FRB180812.J0112+80 & 0.600 & $0.675_{-0.650}^{+0.200}$ & $0.635_{-0.273}^{+0.368}$ & $0.535_{-0.475}^{+1.450}$ & $0.625_{-0.495}^{+1.400}$ & $0.375_{-0.341}^{+1.530}$ \\
\hline FRB180810.J1159+83 & 0.102 & $0.115_{-0.115}^{+0.124}$ & $0.075_{-0.075}^{+0.121}$ & $0.005_{-0.005}^{+0.130}$ & $0.085_{-0.085}^{+0.112}$ & $0.435_{-0.040}^{+0.120}$ \\
\hline FRB180810.J0646+34 & 0.259 & $0.305_{-0.305}^{+0.151}$ & $0.265_{-0.191}^{+1.210}$ & $0.165_{-0.150}^{+1.270}$ & $0.255_{-0.218}^{+1.230}$ & $0.005_{-0.005}^{+0.209}$ \\
\hline FRB 180806.J1515+75 & 0.582 & $0.655_{-0630}^{+0.199}$ & $0.615_{-0.084}^{+0.317}$ & $0.515_{-0.457}^{+1.430}$ & $0.595_{-0471}^{+1.410}$ & $0.365_{-0.333}^{+1.010}$ \\
\hline FRB180801.J2130+72 & 0.472 & $0.535_{-0550}^{+0.185}$ & $0.495_{-0269}^{+1.384}$ & $0.395_{-0.353}^{+1.431}$ & $0.475_{-0.388}^{+1.350}$ & $\begin{array}{r}0.225_{-0.405}^{+0.333} \\
0.450\end{array}$ \\
\hline FRB180730.J0353+87 & 0.660 & $0.745^{+0.212}$ & $0.705^{-0.269}$ & $0.615^{-0.353}+.480$ & $0.695^{-1.450}$ & $\begin{array}{r}-0.205 \\
0.455_{-0.413}^{+1.560}\end{array}$ \\
\hline FRB180729.J1316+55 & 0.066 & $0.065^{-0.110}$ & $0.035^{-0.326}$ & $0.005^{-0.0 .093}$ & $0.035^{-0.044}$ & $1.325_{-0.000}^{-0.413}$ \\
\hline FRB180729.J0558+56 & 0.185 & $0.215^{-0.065}$ & $0.175^{-0.035}$ & $0.085^{-0.0005}+150$ & $0.175^{-0.1 .180}$ & $0.005^{+0.136}$ \\
\hline FRB180727.J1311+26 & 0.518 & $0.595^{-0.187}$ & $0.555^{-0.1370}$ & $0.455^{-0.085}+.410$ & $0.535^{-0.1 .380}$ & $0.285^{-0.005}$ \\
\hline FRB180725.J0613+67 & 0537 & $0.615^{-0.580}$ & $\begin{array}{l}-0.274 \\
5^{+1.380}\end{array}$ & $\begin{array}{r}-0.405 \\
65^{+1.420}\end{array}$ & $545^{+1.370}$ & $\begin{array}{l}0.20 J_{-0.260} \\
0.315^{+1.490}\end{array}$ \\
\hline FRP180525 & $\begin{array}{l}0.551 \\
0.908\end{array}$ & $\begin{array}{l}-0.201 \\
5+0.155\end{array}$ & - & $\begin{array}{l}0.70-0.413 \\
0.280\end{array}$ & $\begin{array}{l}0.436 \\
0.5+505+1.250\end{array}$ & $0.01 J_{-0.288}$ \\
\hline FRB180525 & 0.298 & $0.345_{-0.345}^{+0.155}$ & $0.295_{-0.229}^{+0.219}$ & $0.205_{-0.187}^{+1.280}$ & $0.285_{-0.241}^{+1.250}$ & $0.015_{-0.015}^{+0.231}$ \\
\hline FRB180515 & 0.269 & $0.315_{-0.315}^{+0.152}$ & $0.275_{-0.197}^{+1.230}$ & $0.165_{-0.153}^{+0.266}$ & $0.265_{-0.226}^{+1.230}$ & $0.005_{-0.005}^{+0.218}$ \\
\hline FRB180430 & 0.082 & $0.085_{-0.085}^{+0.116}$ & $0.045_{-0.045}^{+0.116}$ & $0.005_{-0.005}^{+0.105}$ & $0.055_{-0.055}^{+0.110}$ & $0.845_{-0.010}^{+0.116}$ \\
\hline FRB180324 & 0.301 & $0.355_{-0.355}^{+0.055}$ & $0.305_{-0.230}^{+0.227}$ & $0.205_{-0.186}^{+1.300}$ & $0.295_{-0.248}^{+1.260}$ & $0.005_{-0.005}^{+0.257}$ \\
\hline FRB180315 & 0.369 & $0.425_{-0410}^{+0.170}$ & $0.385_{-0.247}^{+1.290}$ & $0.285_{-0.257}^{+0.1830}$ & $0.365_{-0.303}^{+1.240}$ & $0.095_{-0.095}^{+0.0253}$ \\
\hline FRB180212 & 0.112 & $0.125^{+0.126}$ & $0.085_{-0.085}^{-0.241}$ & $0.015_{-0.015}^{-0.231}$ & $0.085_{-0.085}^{+0.303}$ & $0.335_{-0.040}^{+0.095}$ \\
\hline FRB 180131 & 0.515 & $0.585^{-0.187}$ & $0.545_{-0.273}^{+1.370}$ & $0.445_{-0.396}^{-0.013}$ & $0.525_{-0.425}^{-1.085}$ & $0.285_{-0261}^{+0.040}$ \\
\hline FRB180130 & 0.254 & $0.295^{-0.150}$ & $0.255^{-1.220}$ & $0.155_{-0.141}^{-0.396}$ & $0.245_{-0210}^{-0.423}$ & $0.005^{-0.199}$ \\
\hline FRB180128.2 & 380 & $0.435^{-0.171}$ & $\begin{array}{r}-0.186 \\
5^{+1.290}\end{array}$ & $0.295^{-0.1 .310}$ & $0.375^{-0.2100}$ & $0.125^{-0.005}$ \\
\hline FRB180128.0 & 0 & $5^{+0.168}$ & $15^{+0.348}$ & $\begin{array}{l}-0.266 \\
5^{+1.310}\end{array}$ & $35^{+1.270}$ & $\begin{array}{r}-0.125 \\
0.065^{+0.236}\end{array}$ \\
\hline & & $\begin{array}{l}0.50 J_{-0.370} \\
255+0.155\end{array}$ & $\begin{array}{l}0.045-0.229 \\
0.305+0.227\end{array}$ & & & $\begin{array}{r}0.00 J_{-0.065} \\
0.005+0.257\end{array}$ \\
\hline FRB180119 & 0.306 & $0.355_{-0.355}^{+0.153}$ & $0.305_{-0.230}^{+0.227}$ & $0.205_{-0.186}^{+1.300}$ & $0.295_{-0.248}^{+1.260}$ & $0.005_{-0.005}^{+0.25 I}$ \\
\hline FRB180110 & 0.565 & $0.635_{-0.610}^{+0.199}$ & $0.605_{-0.276}^{+1.390}$ & $0.495_{-0.439}^{+1.430}$ & $0.575_{-0.457}^{+1.400}$ & $0.335_{-0.305}^{+1.510}$ \\
\hline FRB171216 & 0.138 & $0.155_{-0.155}^{+0.132}$ & $0.115_{-0.115}^{+0.128}$ & $0.035_{-0.035}^{+0.140}$ & $0.115_{-0.108}^{+0.168}$ & $0.095_{-0.040}^{+0.158}$ \\
\hline FRB 171213 & 0.102 & $0.115_{-0.115}^{+0.124}$ & $0.075_{-0.075}^{+0.121}$ & $0.005_{-0.005}^{+0.130}$ & $0.085_{-0.085}^{+0.112}$ & $0.435_{-0.040}^{+0.120}$ \\
\hline FRB171116 & 0.485 & $0.555_{-0.540}^{+0.186}$ & $0.515_{-0.270}^{+1.340}$ & $0.415_{-0.371}^{+1.390}$ & $0.505_{-0413}^{+1.350}$ & $0.245_{-0.224}^{+1.480}$ \\
\hline FRB171020 & 0.063 & $0.065_{-0.065}^{+0.110}$ & $0.035_{-0.035}^{+0.101}$ & $0.005_{-0.005}^{+0.093}$ & $0.035_{-0.035}^{+0.108}$ & $1.325_{-0.000}^{+0.107}$ \\
\hline FRB171019 & 0.353 & $0.405_{-0.390}^{-0.063}$ & $0.365_{-0.238}^{-0.035}$ & $0.265_{-0240}^{-0.003}$ & $0.345_{-0287}^{-0.035}$ & $0.095_{-0.005}^{+0.000}$ \\
\hline FRB171004 & 0.222 & $0.255^{+0.146}$ & $0.215^{+0.266}$ & $0.115^{+0.161}$ & $0.205^{+1.190}$ & $0.005^{+0.164}$ \\
\hline FRB 171003 & 0.353 & $0.405^{-0.255}$ & $0.365^{-0.1264}$ & $0.265^{+1.300}$ & $0.345^{+1.290}$ & $0.095^{+0.226}$ \\
\hline FRB170906 & 93 & $0.345^{+0.155}$ & $0.295^{-0.238}+0.215$ & $\begin{array}{l}-0.240 \\
0.205^{+1.280}\end{array}$ & $0.285^{-0.287}+250$ & $0.015^{-0.095}$ \\
\hline & & & & $0.205-0.187$ & $0.20 J_{-0.241}$ & \\
\hline FRB170827 & 0.117 & $0.125_{-0.125}^{+0.126}$ & $0.085_{-0.085}^{+0.123}$ & $0.015_{-0.015}^{+0.130}$ & $0.085_{-0.085}^{+0.123}$ & $0.335_{-0.040}^{+0.129}$ \\
\hline
\end{tabular}


Table B.1. continued.

\begin{tabular}{|c|c|c|c|c|c|c|}
\hline FRB & $z_{\mathrm{s}}($ conventional $)$ & $z_{\mathrm{s}}($ model a) & $z_{\mathrm{s}}($ model b) & $z_{\mathrm{s}}($ model $\mathrm{c})$ & $z_{\mathrm{s}}($ model d $)$ & $\overline{z_{\mathrm{s}}(\operatorname{model~} \mathrm{e})}$ \\
\hline FRB170712 & 0.229 & $0.265_{-0.265}^{+0.147}$ & $0.225_{-0.167}^{+1.200}$ & $0.135_{-0.125}^{+1.240}$ & $0.215_{-0.185}^{+1.210}$ & $0.005_{-0.005}^{+0.173}$ \\
\hline FRB 170707 & 0.166 & $0.185_{-0.185}^{+0.137}$ & $0.145_{-0.139}^{+0.147}$ & $0.055_{-0.055}^{+0.150}$ & $0.145_{-0.127}^{+1.170}$ & $0.005_{-0.005}^{+0.133}$ \\
\hline FRB 170428 & 0.793 & $0.895_{-0.860}^{+0.226}$ & $0.855_{-0.330}^{+0.277}$ & $0.745_{-0.648}^{+1.560}$ & $0.835_{-0.644}^{+1.530}$ & $0.605_{-0.544}^{+1.620}$ \\
\hline FRB170416 & 0.403 & $0.465_{-0.450}^{+0.172}$ & $0.425_{-0.262}^{+1.300}$ & $0.315_{-0.283}^{+1.330}$ & $0.405_{-0.333}^{+1.310}$ & $0.155_{-0.155}^{+0.225}$ \\
\hline FRB170107 & 0.479 & $0.545_{-0.530}^{+0.186}$ & $0.505_{-0.269}^{+1.350}$ & $0.405_{-0.362}^{+1.390}$ & $0.485_{-0.395}^{+1.340}$ & $0.245_{-0.224}^{+1.460}$ \\
\hline FRB 160608 & 0.370 & $0.425_{-0.410}^{+0.530}$ & $\begin{array}{r}-0.385_{-0.247}^{+1.269} \\
0.09\end{array}$ & $0.285_{-0.257}^{+0.362}$ & $\begin{array}{r}0.365_{-0.303}^{-0.393} \\
.300\end{array}$ & $0.095_{-0.095}^{-0.224}$ \\
\hline FRB 160410 & 0.184 & $0.215_{-0.215}^{+0.410}$ & $0.175_{-0.151}^{+0.177}$ & $0.085_{-0.085}^{+0.150}$ & $0.175_{-0.153}^{+0.303}$ & $0.005_{-0.005}^{+0.136}$ \\
\hline FRB160317 & 0.705 & $\begin{array}{r}-0.215 \\
0.795_{-0.0760}^{+0.213}\end{array}$ & $\begin{array}{r}-0.151 \\
0.755_{-0.289}^{+0.306}\end{array}$ & $\begin{array}{r}-0.085 \\
0.635_{-0.559}^{+1.520}\end{array}$ & $\begin{array}{r}-0.153 \\
0.735_{-0.572}^{+1.480}\end{array}$ & $\begin{array}{r}-0.005 \\
0.495_{-0.447}^{+1.580}\end{array}$ \\
\hline FRB 160102 & 2.153 & $2.565_{-0.383}^{+0 . / 613}$ & $2.535_{-0.410}^{+0.422}$ & $2.415_{-0.513}^{+1.960}$ & $2.495_{-0.454}^{+1.920}$ & $2.345_{-0.611}^{+0.447}$ \\
\hline FRB 151230 & 0.769 & $0.865_{-0.830}^{+0.383}$ & $0.825_{-0.360}^{-0.410}$ & $0.715_{-0.628}^{+1.550}$ & $0.795_{-0.611}^{+1.510}$ & $0.575_{-0.518}^{+0.011}$ \\
\hline FRB151206 & 1.458 & $1.655_{-1.550}^{+0.303}$ & $1.625_{-0.401}^{+0.331}$ & $1.505_{-0.638}^{+1.770}$ & $1.585_{-0.483}^{+1.730}$ & $1.415_{-0.735}^{+1.810}$ \\
\hline FRB150807 & 0.191 & $0.215_{-0.215}^{+0.141}$ & $0.175_{-0.151}^{+0.177}$ & $0.085_{-0.085}^{+0.030}$ & $0.175_{-0.153}^{+1.180}$ & $0.005_{-0.005}^{+0.136}$ \\
\hline FRB150610 & 1.227 & $1.395_{-0.306}^{+0.287}$ & $1.355_{-0.364}^{+0.312}$ & $1.245_{-0.673}^{+1.690}$ & $1.335_{-0.542}^{+0.1 .653}$ & $1.125_{-0.655}^{+1.003}$ \\
\hline FRB 150418 & 0.490 & $0.555_{-0.540}^{+0.500}$ & $0.515_{-0.270}^{+1.344}$ & $0.415_{-0.371}^{+1.390}$ & $0.505_{-0.413}^{+1.350}$ & $0.245_{-0.224}^{+0.033}$ \\
\hline FRB 150215 & 0.565 & $0.635_{-0.610}^{+0.540}$ & $0.605_{-0.276}^{+1.390}$ & $0.495_{-0.439}^{+1.430}$ & $0.575_{-0.457}^{+0.400}$ & $0.335_{-0.305}^{-0.224}$ \\
\hline FRB141113 & 0.177 & $0.205_{-0.205}^{+0.610}$ & $0.165_{-0.148}^{+0.166}$ & $0.075_{-0.075}^{+0.439}$ & $0.165_{-0.145}^{+1.170}$ & $0.005_{-0.005}^{+0.132}$ \\
\hline FRB140514 & 0.440 & $0.495_{-0.480}^{+0.185}$ & $0.455_{-0.280}^{+0.148}$ & $0.355_{-0.318}^{+1.370}$ & $0.445_{-0.366}^{+0.1430}$ & $0.195_{-0.382}^{-0.005}$ \\
\hline FRB131104 & 0.590 & $0.665_{-0640}^{+0.180}$ & $0.625_{-0.278}^{+0.280}$ & $0.515_{-0456}^{+0.418}$ & $\begin{array}{r}-0.000 \\
0.595_{-0446}^{+1.420}\end{array}$ & $0.365_{-0.332}^{+1.520}$ \\
\hline FRB130729 & 0.692 & $\begin{array}{r}-0.640 \\
0.775_{-0.213}^{+0.213}\end{array}$ & $\begin{array}{r}-0.278 \\
0.735_{-0.289}^{+0.289}\end{array}$ & $\begin{array}{r}-0.456 \\
0.635_{-0.562}^{+1.480}\end{array}$ & $0.715_{-0.458}^{+0.468}$ & $0.485_{-0.440}^{+0.532}$ \\
\hline FRB 130628 & 0.348 & $\begin{array}{r}0.395_{-0.380}^{+0.160} \\
\end{array}$ & $\begin{array}{r}-0.302 \\
0.355_{-0.233}^{+1.280}\end{array}$ & $\begin{array}{r}-0.562 \\
0.265_{-0.240}^{+1.310}\end{array}$ & $\begin{array}{r}-0.558 \\
0.335_{-0.279}^{+1.290}\end{array}$ & $\begin{array}{l}-0.440 \\
0.075_{-0.075}^{+0.236}\end{array}$ \\
\hline FRB 130626 & 0.738 & $0.835_{-0.250}^{+0.220}$ & $0.795_{-0.269}^{+0.358}$ & $0.685_{-0.606}^{+1.530}$ & $0.775_{-0.601}^{+1.470}$ & $0.535_{-0.483}^{+1.580}$ \\
\hline FRB121102 & 0.307 & $0.355_{-0.355}^{+0.155}$ & $0.305_{-0.230}^{+0.227}$ & $0.205_{-0.186}^{+1.300}$ & $0.295_{-0.248}^{+1.260}$ & $0.005_{-0.005}^{+0.257}$ \\
\hline FRB121002 & 1.296 & $1.475_{-0.300}^{+0.302}$ & $1.435_{-0.362}^{+0.322}$ & $1.315_{-0.663}^{+1.1720}$ & $1.395_{-0507}^{+1.690}$ & $1.215_{-0.688}^{+1.750}$ \\
\hline FRB120127 & 0.435 & $0.495_{-0.480}^{+0.184}$ & $0.455_{-0.280}^{+0.288}$ & $0.355_{-0.318}^{+1.370}$ & $0.445_{-0.366}^{+1.330}$ & $0.195_{-0.182}^{+0.331}$ \\
\hline FRB 110703 & 0.893 & $1.005_{-0.960}^{+0.240}$ & $0.975_{-0.273}^{+0.280}$ & $0.855_{-0.652}^{+1.610}$ & $0.945_{-0.658}^{+0.500}$ & $0.725_{-0.581}^{-0.182}$ \\
\hline FRB110626 & 0.563 & $0.635_{-0.610}^{+0.199}$ & $0.605_{-0.276}^{+1.390}$ & $0.495_{-0.439}^{+1.430}$ & $0.575_{-0.457}^{+1.030}$ & $0.335_{-0.305}^{-1.510}$ \\
\hline FRB 110523 & 0.483 & $0.545_{-0.530}^{+0.610}$ & $0.505_{-0.269}^{+.1 .350}$ & $\begin{array}{r}-0.439 \\
0.405_{-0.362}^{+1.390}\end{array}$ & $0.485_{-0.395}^{+0.457}$ & $\begin{array}{r}-0.305 \\
0.245_{-0.224}^{+1.460}\end{array}$ \\
\hline FRB 110220 & 0.758 & $0.855_{-0230}^{+0.228}$ & $0.805_{-0.387}^{-0.269}$ & $0.715_{-0632}^{-0.562}$ & $0.785_{-0660}^{-0.595}$ & $0.565_{-0.510}^{+1.590}$ \\
\hline FRB110214 & 0.115 & $0.125_{-0125}^{-0.126}$ & $\begin{array}{r}-0.387 \\
0.085_{-0.085}^{+0.123}\end{array}$ & $\begin{array}{r}-0.632 \\
0.015_{-0.015}^{+0.130}\end{array}$ & $0.085_{-0.085}^{-0.606}$ & $\begin{array}{l}-0.510 \\
0.335_{-0.040}^{+0.129}\end{array}$ \\
\hline FRB090625 & 0.723 & $0.815^{-0.214}$ & $0.775^{-0.0 .327}$ & $0.675^{-0.015}+10$ & $\begin{array}{r}-0.085 \\
0.755^{+1.480}\end{array}$ & $0.525_{-0.475}^{+1.570}$ \\
\hline FRB010724 & 0.275 & $0.325_{-0.153}^{-0.780}$ & $\begin{array}{r}-0.278 \\
0.275_{-0.197}^{+0.198}\end{array}$ & $0.185_{-0169}^{+0.598}$ & $0.265_{-0.234}^{-0.587}$ & $0.005_{-0.005}^{-0.475}$ \\
\hline FRB010621 & 0.185 & $0.215^{+0.141}$ & $\begin{array}{r}-0.228 \\
0.175^{+0.177}\end{array}$ & $\begin{array}{r}-0.169 \\
0.085^{+0.150}\end{array}$ & $0.175^{+1.180}$ & $\begin{array}{l}0.005^{-0.005} \\
0.005^{+0.136}\end{array}$ \\
\hline FRB010321 & 0.947 & $1.065^{+0.242}$ & $1.025^{+0.285}$ & $\begin{array}{l}0.005^{-0.085} \\
0.905^{+1.630}\end{array}$ & $0.995^{+1.590}$ & $\begin{array}{l}0.00 J_{-0.005} \\
0.785^{+1.700}\end{array}$ \\
\hline FRB010125 & 0.567 & $\begin{array}{r}-1.020 \\
0.635_{-0.610}^{+0.199}\end{array}$ & $\begin{array}{r}-0.345 \\
0.605_{-0.390}^{+1.390}\end{array}$ & $\begin{array}{r}-0.650 \\
0.495_{-0.439}^{+1.430}\end{array}$ & $\begin{array}{r}-0.622 \\
0.575_{-0.457}^{+1.400}\end{array}$ & $0.335_{-0305}^{+0.581}$ \\
\hline
\end{tabular}

